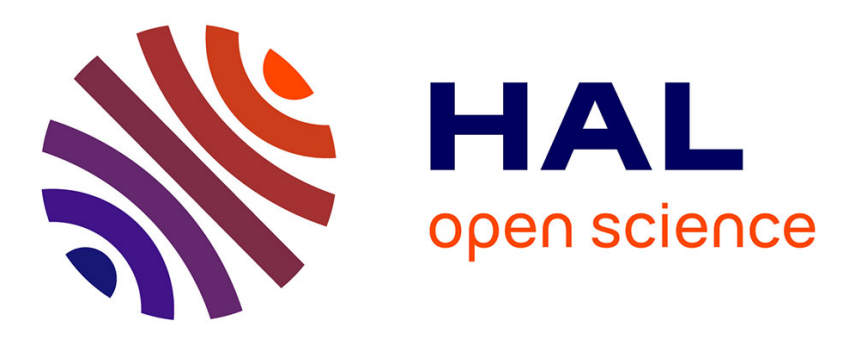

\title{
Constant-Envelope Multicarrier Waveforms for Millimeter Wave 5G Applications
}

Talha Faizur Rahman, Claudio Sacchi, Simone Morosi, Agnese Mazzinghi, Nicola Bartolomei

\section{- To cite this version:}

Talha Faizur Rahman, Claudio Sacchi, Simone Morosi, Agnese Mazzinghi, Nicola Bartolomei. Constant-Envelope Multicarrier Waveforms for Millimeter Wave 5G Applications. IEEE Transactions on Vehicular Technology, 2018, 67 (10), pp.9406 - 9420. 10.1109/TVT.2018.2854723 . hal-01928147

HAL Id: hal-01928147 https://hal-univ-rennes1.archives-ouvertes.fr/hal-01928147

Submitted on 14 Dec 2018

HAL is a multi-disciplinary open access archive for the deposit and dissemination of scientific research documents, whether they are published or not. The documents may come from teaching and research institutions in France or abroad, or from public or private research centers.
L'archive ouverte pluridisciplinaire $\mathbf{H A L}$, est destinée au dépôt et à la diffusion de documents scientifiques de niveau recherche, publiés ou non, émanant des établissements d'enseignement et de recherche français ou étrangers, des laboratoires publics ou privés. 


\title{
Constant-Envelope Multicarrier Waveforms for Millimeter Wave 5G Applications
}

\author{
Talha Faizur Rahman, Claudio Sacchi, Senior Member, IEEE, Simone Morosi, Member, IEEE, Agnese Mazzinghi, \\ and Nicola Bartolomei
}

\begin{abstract}
A key point of Fifth Generation (5G) wireless networking will be the exploitation of higher frequency bands in the millimeter wave ( $\mathrm{mm}$-Wave) spectrum to provide unprecedented data rates to mobile users. In such a perspective, the PHYsical (PHY) layer design priorities should be reconsidered. In this paper, we investigate Constant-Envelope Multicarrier (CEMC) waveforms for future adoption in mm-Wave $5 \mathrm{G}$ transmissions, namely: Constant-Envelope Orthogonal Frequency Division Multiplexing (CE-OFDM) and Constant-Envelope SingleCarrier OFDM (CE-SC-OFDM). These waveforms are obtained by imposing a nonlinear phase modulation to a real-valued OFDM and SC-OFDM signal, respectively. Thanks to their OdB Peak-to-Average-Power Ratio (PAPR), such unconventional signaling formats are insensitive to nonlinear distortions and allow to exploit the flexibility of conventional multicarrier systems together with augmented resilience against multipath fading and phase noise. CE-OFDM and CE-SC-OFDM have been assessed by means of computer simulations in a short-range mm-Wave 5G scenario, i.e. downlink transmission in outdoor picocells. Simulation results demonstrate that $\mathrm{CE}$ multicarrier waveforms enhance robustness and increase coverage and capacity in the proposed scenario, as compared to conventional OFDM and SCOFDM counterparts.
\end{abstract}

Index Terms-5G, mm-Wave communications, multicarrier modulations, SIW antennas.

\section{INTRODUCTION}

$\mathrm{T}$ He exploitation of mm-Wave bands will be one of the basic pillars for $5 \mathrm{G}$ mobile broadband networking. Thanks to the huge availability of mm-Wave spectrum $(71-76 \mathrm{GHz}$ and 81-86 GHz in the licensed E-band) and the constant decrease of the cost of high-frequency electronics components, it has already been possible to implement transceivers capable of supporting unprecedented data rates of the order of some Gbps. However, there are some concerns about the transmission characteristics at such high frequencies. As claimed in [1],

T. F. Rahman is with Center for Advanced Studies in Telecommunication (CAST), COMSATS, Institute of Information Technology, 45550, Islamabad, Pakistan (e-mail: talha.rahman@alumni.unitn.it)

C. Sacchi is with the DISI of University of Trento, 38123, Trento, (Italy), and with the Consorzio Nazionale Interuniversitario per le Telecomunicazioni (CNIT), 43124, Parma (Italy), (e-mail: claudio.sacchi@unitn.it)

S. Morosi and A. Mazzinghi are with the University of Florence, Dept. of Information Engineering (DINFO), 50139, Florence, Italy, and with the Consorzio Nazionale Interuniversitario per le Telecomunicazioni (CNIT) 43124, Parma (Italy) (e-mail: simone.morosi@unifi.it, agnese.mazzinghi@unifi.it)

N. Bartolomei is with the IETR of University of Rennes I, Rennes, France (e-mail: nicola.bartolomei@univ-rennes1.fr)

Paper submitted on: Dec. 28, 2017, revised on: March 30, 2018, May 15, 2018, and June 15, 2018. A preliminary version of this paper has been published at the ET-5G Workshop, Washington DC, 4-8 Dec. 2016. the reduced size of future 5G small cells (max. 150 meters of inter-cell distance) will limit oxygen absorption and rain fading into acceptable ranges. However, the pathloss is still an issue if compared to that of lower frequency bands used in 4G. Moreover, the shadowing noticeably impacts on the link availability, particularly in case of Non-Line-of-Sight (NLOS) propagation [2]. From the considerations drawn above, the power efficiency of the transmission system is a requirement tendentially more stringent than spectral efficiency. In such a framework, the waveform design plays a key role. It should be pointed out that mm-Wave power amplifiers characterized by high power gain whereas satisfactory Power-Added Efficiency (PAE) are generally nonlinear with saturating Amplitude-to-Amplitude (AM/AM) characteristics. The full exploitation of the available $\mathrm{RF}$ power resources drives the amplifier to saturation. If the modulated waveform is characterized by high Peak-to-Average Power Ratio (PAPR), the amplifier saturation would involve huge amplitude distortion and considerable spectral regrowth. In order to avoid nonlinear distortion effects, we should use constant-amplitude signaling formats or drive the amplifier back from saturation by imposing a significant back-off to the transmitted power. The recent wireless transmission standards have considered the use of multicarrier modulations, namely: OFDM and DFT-precoded OFDM (also known as SingleCarrier (SC)-OFDM or, in the multi-user case, SC-FDMA [3]). These waveforms offer advantages in terms of resilience against frequency-selective multipath propagation and flexible orthogonal multiple access. As they are obtained by means of an IDFT operation, state-of-the-art multicarrier waveforms are generally affected by high PAPR. 99.9-percentile PAPR comparative results shown in [3] (Ch.7, Tab. 7.1, pp. 132) for multicarrier-based orthogonal multiple access systems indicate the highest value $(11.1 \mathrm{~dB})$ for OFDMA. The PAPR of SCFDMA mainly depends on the employed subcarrier allocation methodology (Interleaved, Localized or Distributed FDMA [3]). Although, in all the cases, the PAPR of SC-FDMA is lower than that of OFDMA, the achieved values are still not negligible, as LFDMA and DFDMA show PAPR higher than $7 \mathrm{~dB}$, whereas PAPR in IFDMA depends on modulation constellation and pulse shaping. The application of Input Backoff (IBO) may thus be necessary to limit the nonlinear distortion effects. Unfortunately, the backoff does not only reduce the Carrier Power-to-Noise Ratio (CNR), but also lowers the PAE with a consequential increase of power consumption [4].

Despite the aforesaid issues, most recent works have confirmed a general consensus about the use of multicarrier modulations also in 5G [1] [5], but with some improvements w.r.t. the conventional formats. Therefore, instead of OFDM 
we usually speak of "OFDM-inspired" waveforms [5]. These are basically: Filter-Bank Multicarrier modulation (FBMC), Generalized Frequency Division Multiplexing (GFDM) and Universal Filtered Multicarrier modulation (UFMC) [5]. Such waveforms are aimed at preserving the advantages of basic multicarrier modulations, while overcoming some throughput limitations due to long cyclic prefix insertion and spectrum leakage. Indeed, the "OFDM-inspired" waveform design is mainly driven by the necessity of optimizing the exploitation of the scarce resources provided by $s u b-6 \mathrm{GHz}$ frequency bands. We think that such a design philosophy does not reflect the claimed objectives of the " $5 G$ vision" thoroughly described in [6], where fiber-like user experience with up to $10 \mathrm{~Gb} / \mathrm{s}$ data rates are anticipated to support mobile cloud services and immersive virtual reality applications. It is stated in [6] that at least $1 \mathrm{GHz}$ of supplementary spectrum is required to achieve such visionary targets. The mm-Wave bandwidth portions can provide this necessary amount of radio resources.

In this paper, we propose two waveform solutions for mmWave $5 \mathrm{G}$ transmission that are not only able at preserving some key advantages of multicarrier modulations, but also solving the problems inherent to the inefficient exploitation of power resources. The assessed waveforms are: ConstantEnvelope OFDM (CE-OFDM), originally proposed in [7], and Constant-Envelope Single-Carrier OFDM (CE-SC-OFDM), whose multi-user version (CE-SCFDMA) has been presented in [8]. Both of them resort to a non-linear phase modulation applied to real-valued normalized multicarrier signal. The stand out features of such waveforms are the following:

- Fixed OdB PAPR: the signal can be transmitted through saturating amplifiers without amplitude distortion and spectral regrowth;

- As claimed in [9], CE-MC signals can take advantage of the correlation between subcarriers, as inherent diversity effect caused by the intermodulation, which results from the nonlinear phase modulation of the real-valued multicarrier signals. Therefore, the advantages of multicarrier modulations are still maintained together with augmented diversity against multipath fading;

- CE multicarrier signals are more resilient to phase noise effects than conventional multicarrier counterparts. This is because the phase noise becomes additive after the phase demodulation [10].

The price to be paid in order to gain the aforesaid competitive advantages is an increased bandwidth occupation of the $\mathrm{RF}$ signal. This issue is inherent to the double-sided spectral shape of real-valued OFDM and SC-OFDM signals that are fed to the phase modulator. Considering these aspects in sight, we peform waveform assessment in two steps:

- Link performance evaluation in terms of Bit-Error-Rate (BER) in the presence of mm-Wave multipath propagation, nonlinear distortion and phase noise.

- Coverage analysis for CE multicarrier waveforms in specific 5G application scenario, namely: a downlink picocell transmission operating at $73 \mathrm{GHz}$. The link budget of the considered scenario will be computed on the basis of large scale propagation phenomena (pathloss and shadowing) along with a specific RF design, characterized by precise power constraints. To this aim, a Substrate Integrated Waveguide (SIW) slotted antenna array with squared cosecant pattern is proposed and designed in order to implement an efficient, realistic and cost-effective RF solution.

The paper is structured as follows: Section II will review the state-of-the-art about the candidate waveforms for $5 \mathrm{G}$ applications and will highlight the innovation yielded by the present work. In Section III, CE waveforms will be described in terms of transmitted signals analysis and detection methodologies. They will then be compared with other state-of-the-art waveforms considered for $5 \mathrm{G}$ in the framework of $\mathrm{mm}$-Wave urban transmission, providing a preliminary taxonomy of the different techniques. Section IV focuses on the description of the $5 \mathrm{G}$ application scenario chosen for performance evaluation. Section V will present and discuss simulation results. Paper conclusions are finally drawn in Section VI.

\section{BACKGROUND AND INNOVATION}

\section{A. State-of-the-art overview: $5 G$ candidate waveforms}

Despite the well known issues, OFDM and other multicarrier modulations are still occupying the pole position in the race to become $5 \mathrm{G}$ multiple access technique, as clearly stated in the white paper recently published by Rohde \& Schwarz GmbH \& Co (D) [11]. Reading the notes of [11], it seems that the flexibility inherent to orthogonal multiple access represents an indispensable feature also for future $5 \mathrm{G}$ mobile communications. Nevertheless, some authors are considering, as alternative, non-orthogonal access options [12]. Non-Orthogonal Multiple Access (NOMA) is one such technique that allows to improve system spectral efficiency as the restrictions on radio resource allocation, needed to guarantee the access orthogonality, can be avoided [12]. However, NOMA requires more complex receivers that are also in charge of interference cancellation. Moreover, as stated in [12], the outage performance of NOMA critically depends on the choice of targeted data rates and allocated power.

In the recent work of Gerzaguet et. al. [13], a comparison on main 5G candidate waveforms has been carried out, considering the transmission of information over sub- $6 \mathrm{Ghz}$ bandwidths. All the considered techniques are based on the multicarrier concept and allow orthogonal multiple access, namely: OFDM, SC-OFDM, FBMC, UFMC and GFDM.

FBMC is based on the transmission of parallel data streams with the help of IDFT processing followed by bank of modulated filters. Specifically, the output of each IDFT branch is sent to a prototype filter that is chosen to have very low adjacent channel leakage. Various implementations of FBMC are considered in practice, differentiated on the basis of chosen digital modulation format (see [13] for further details). FBMC waveforms eliminate the need for $\mathrm{CP}$ by means of transmit-receive prototype filter bank and one-tap equalization performed at receiver. The FBMC spectral efficiency is improved w.r.t. OFDM and SC-OFDM, however, the complexity of transmitter and receiver (TX/RX) increases thanks to the presence of narrowband highly-selective prototype filters that 
are usually implemented in the digital domain by means of FIR structures. UFMC is derived from OFDM waveform combined with post-filtering through which a group of subcarriers is filtered in the frequency domain [14]. Such a filtering operation leads to reduced out-of-band leakage which is, otherwise, present as in the case of conventional OFDM. However, the spectral efficiency reduces due to long tail of shaping filters. Despite the inefficiency of bandwidth utilization, the overall complexity of UFMC is consistently reduced as compared to that of FBMC. GFDM is another multicarrier system that digitally implements the classical filter band approach [15]. Multiple parallel QAM-modulated data streams are aided with CP. After CP insertion, digital pulse shaping is performed subcarrier-wise together with tail biting techniques being applied to shorten the $\mathrm{CP}$ overhead in order to improve spectral efficiency.

The comparative analysis of [13] shows better results achieved in terms of reduced adjacent channel leakage by UFMC and FBMC, while the poorer are for GFDM and OFDM. As far as the computational complexity of TX/RX chain is concerned, conventional OFDM provides the lowest complexity with GFDM has compuational burden comparable to that of OFDM. On the other hand, FBMC and UFMC exhibit a considerably higher complexity due to involvement of subcarrier filtering operations. The PAPR analysis for the assessed waveforms shows the best results for SC-OFDM (around $7.5 \mathrm{~dB}$ ), while the rest have almost the same high PAPR (around $10.5 \mathrm{~dB}$ ). This implies that in the presence of nonlinear amplification, SC-OFDM gets the advantage of requiring reduced $\mathrm{IBO}$, while the other $5 \mathrm{G}$ waveform candidates will require higher IBO in order to attain acceptable link performance.

\section{B. Contribution of the paper}

The waveform design proposed by the literature importantly considers the maximization of spectral efficiency and the frequency agility. Indeed, as remarked by [13], the target of these waveforms is to exploit existing underutilized fragmented spectrum in the $s u b-6 \mathrm{GHz}$ frequency bands. However, $\mathrm{mm}$-Wave transmission requires extra attention from engineers in order to address new priorities. First of all, the efficient exploitation of the power resources is a "must" in the mmWave spectrum band. The pathloss and shadowing are considerably huge, in particular when NLOS propagation scenarios are considered [2]. The waveforms assessed in [13] are all characterized by high PAPR and require considerable power back-offs for nonlinear amplifiers. The vulnerability to phase noise is another critical aspect in multicarrier modulations. As shown in the detailed analysis of [16], phase noise can compromise subcarrier orthogonality by introducing InterChannel Interference (ICI) at the receiver.

We believe that these two issues can be efficiently solved by adopting a different waveform design. In few words, we are seeking for waveforms that can preserve the nonquestionable advantages of multicarrier in terms of flexibility and orthogonal multiple access efficiency, while avoiding the capacity reduction due to the presence of nonlinear distortions and phase noise. Constant-Envelope (CE) multicarrier modulations, namely: CE-OFDM [7] and CE-SC-OFDM [8] seem to cope quite well with the aforesaid requirements. CE multicarrier signals have PAPR identically equal to $0 \mathrm{~dB}$, thus allowing IBO-free transmission. Moreover, they exhibit improved robustness against frequency-selective multipath fading thanks to the augmented frequency diversity yielded by nonlinear phase modulation [8] [9]. Finally, as shown in [10], CE multicarrier waveforms offer better performance than conventional OFDM-based counterparts in the presence of high-frequency noisy oscillators [10]. This is primarily due to phase modulation characteristics of the signal that considers phase noise as an additive impairment.

The drawback of CE-OFDM is related to the throughput reduction of (at least) $50 \%$ w.r.t. conventional OFDM, mainly because the double-sided spectrum of real-valued IDFT employed in CE multicarrier signaling. However, if we consider the presence of frequency-selective multipath fading and nonlinear amplification, the scenario completely changes in favor of CE multicarrier waveforms, as clearly shown in [7] and [8]. We believe that such considerations fully motivate the enrollment of these signalling formats in the competition for $5 \mathrm{G}$ waveform selection.

In the recent literature, some papers have been published, dealing with practical applications of CE-OFDM in highly differentiated communication and networking contexts. In [17], it has been used as alternative to OFDM in radar-based target detection. In [18] and [19], it has been investigated for short-reach multimode fiber links and impulsive noise power-line communication channels. The investigation of this waveform for mm-Wave transmission is very recent with few published work. In [20], Magueta et. al proposed a new spacefrequency equalizer for MIMO CE-OFDM transmission over mm-Wave multipath channels. The adoption of CE-OFDM for broadband mm-Wave $5 \mathrm{G}$ transmission systems has been preliminary discussed in [21] by the same authors of this paper. The present paper can be thus regarded as an extension of [21], with additional analyses and evaluations are presented to assess the viability of $\mathrm{CE}$ multicarrier waveforms in realistic mm-Wave 5G transmission scenarios. The novel contributions of this work w.r.t. [21] can be summarized as follows:

- Both CE-OFDM and CE-SC-OFDM have been assessed, whereas [21] only dealt with CE-OFDM;

- More insightful details about CE multicarrier waveforms generation, spectral efficiency and trasnsmission/detection have been provided. Critical comparison with other $5 \mathrm{G}$ waveform candidates has been discussed;

- Alternative solutions for performing task of basic arctangent detector have been discussed for phase demodulation in subection II-A (namely: the low-complexity CE multicarrier detectors of [22]) and tested in the simulation trials;

- A deeper comparative link performance analysis has been provided in Section $\mathrm{V}$, including the effects of phase noise, which are neglected in [21];

- The coverage, expressed in terms of BER and goodput availability vs. distance, has been carefully assessed in the picocell downlink scenario by considering the three- 


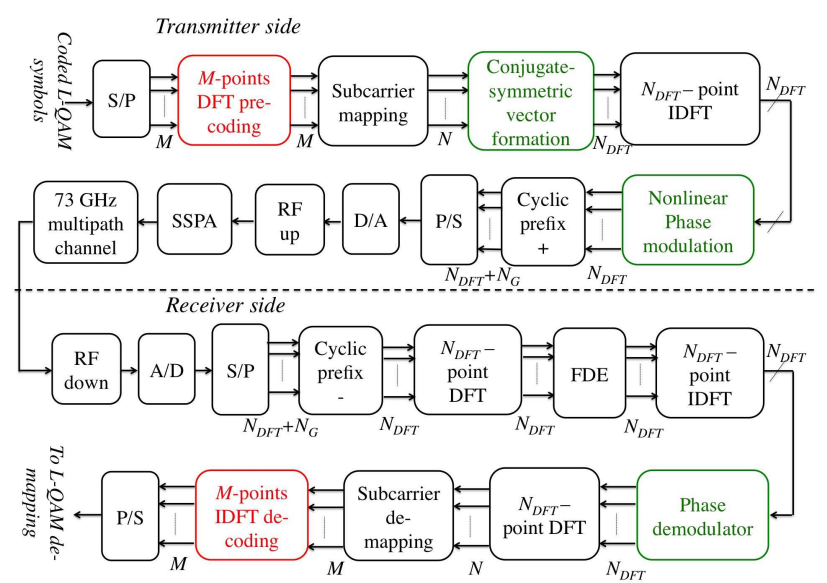

Figure 1. Block diagram of the constant-envelope multicarrier transceiver (black line: OFDM basic blocks, green line: CE-OFDM adjunct blocks, red line: CE-SC-OFDM adjunct blocks)

state channel model of [2]. In [21], such kind of analysis was only roughly sketched. SIW antenna design has been improved w.r.t. that proposed in [21].

Moreover, we emphasize that the added value of this work w.r.t [20] is offered in terms of a complete end-to-end system analysis and performance assessment in the framework of a $\mathrm{mm}$-Wave 5G broadband application scenario. The focus of [20] is rather on a specific space-frequency signal processing technique, aimed at improving the efficiency of MIMO CEOFDM detection in a mm-Wave multipath channel.

\section{Constant-Envelope Multicarrier Waveforms}

\section{A. Constant-Envelope OFDM (CE-OFDM)}

CE-OFDM waveform revolves around nonlinear phase modulation applied to real-valued OFDM signal generated in the baseband digital domain (see Fig. 1). In order to practically obtain the baseband real-valued OFDM signal, a conjugatesymmetric symbol vector is generated every $T$ seconds, starting from $N$ complex L-QAM information symbols $\underline{S}=$ $\left\{S_{1}, S_{2} \cdots, S_{N}\right\}$, where $L$ is an even integer power of two. The output of conjugate-symmetric block $\underline{V}_{c s}$ is given as,

$$
\underline{V}_{c s}=\left[0, S_{1}, \cdots, S_{N}, \underline{Z}_{p}, 0, S_{N}^{*}, \cdots, S_{1}^{*}\right]
$$

The symbol vector $\underline{S}$ is made by $N / M$ symbol blocks, corresponding to the messages transmitted by multiple users of the system, while $\underline{Z}_{p}$ denotes the zero-padding vector of length $N_{z p}$. Without losing generality, we consider the special case of $M=N$, also referred to as single user case. The length of $\underline{V}_{c s}$ is,

$$
N_{D F T}=2(N+1)+N_{z p}=2(N+1) F_{o v}
$$

where $F_{o v}$ is the oversampling factor, defined as, $F_{o v} \triangleq$ $\frac{N_{D F T}}{N_{D F T}-N_{z p}}$ [7]. The oversampling factor should be chosen as a power of 2 . The role of $F_{o v}$ in CE-OFDM waveform formation is critical and will be discussed in the following.

The next step is the computation of the $N_{D F T}$-point IDFT of the complex sequence $\left\{v_{k}=\underline{V}_{c s}[k], k=0, \cdots, N_{D F T}-1\right\}$.
It is easy to verify that such computation provides the realvalued oversampled OFDM sequence as follows [7],

$$
X_{n}=2 \sum_{k=1}^{N}\left[\mathfrak{R}\left\{S_{k}\right\} \cos \left(\frac{2 \pi k n}{N_{D F T}}\right)-\mathfrak{J}\left\{S_{k}\right\} \sin \left(\frac{2 \pi k n}{N_{D F T}}\right)\right]
$$

$\mathfrak{R}\left\{S_{k}\right\}$ and $\mathfrak{J}\left\{S_{k}\right\}$ are, respectively, the real part and the imaginary part of the data symbols $\left\{S_{1}, S_{2} \cdots, S_{N}\right\}$. Afterwards, the nonlinear phase modulation is applied to the realvalued OFDM sequence of (1). The discrete-time sequence, $u_{n}$, obtained at the output of nonlinear phase modulator having $0 \mathrm{~dB}$ PAPR is given as,

$$
u_{n}=e^{j 2 \pi h\left(\Gamma X_{n}\right)}, \quad n=0,1, \cdots, N_{D F T}-1
$$

where $2 \pi h$ is the angular modulation index measured in radians and $\Gamma=\sqrt{\frac{6}{N(L-1)}}$ is the normalization constant, defined in [7]. The cyclic prefix of length $N_{c p}$ is then appended to (4). A Digital-to-Analog (D/A) conversion of the cyclic-prefixed CE-OFDM sequence with sampling rate $1 / T$ is performed. The transmitted CE-OFDM signal is thus given as,

$$
y(t)=A_{c} \mathfrak{R}\left\{e^{j\left[2 \pi f_{0} t+\phi(t)+\vartheta\right]}\right\},-T_{c p} \leq t \leq T
$$

where $A_{c}, f_{0}$, and $\vartheta$ are the amplitude, frequency and phase of carrier signal, respectively, while $\phi(t)=2 \pi h \Gamma X(t)$ is the analog real-valued OFDM signal modulating the carrier phase $\left(X(t)\right.$ is the D/A converted sequence $X_{n}$ of 11$)$. The received baseband CE-OFDM signal can be expressed, after $\mathrm{CP}$ removal, as follows [7],

$$
r(n)=D F T^{-1}\left(H_{k} Y_{k}\right)+w(n), \quad n=0,1, \cdots, N_{D F T}-1
$$

where $H_{k}$ and $Y_{k}$ are the $k^{t h}$ subchannel response and the transmitted CE-OFDM symbol converted in the frequency domain, respectively. The baseband detection of CE-OFDM signals is then performed by means of the following tasks:

- Frequency-domain equalization (FDE), applied to the received signal of (6) as given in (7);

- Phase demodulation applied to the FDE output [7];

$\rho(n)=I_{n}^{\rho}+j Q_{n}^{\rho}=D F T^{-1}\left(\beta_{k} R_{k}\right), \quad n=0,1, \cdots, N_{D F T}-1$

where $\beta_{k}$ is the equalizer response on $k^{\text {th }}$ subchannel and $\left\{R_{k}\right\} \triangleq \operatorname{DFT}(r(n))$. The phase demodulation provides the estimate of the real-valued OFDM sequence as follows,

$$
\hat{X}_{n}=\frac{\phi \hat{(} n)}{2 \pi h \Gamma}, \quad n=0,1, \cdots, N_{D F T}-1
$$

Basically, phase demodulation is performed by means of the arctangent (ARC) operation, namely: $\hat{\phi}(n)=\arctan \left(Q_{n}^{\rho} / I_{n}^{\rho}\right)$ [7]. It should be highlighted that arctangent demodulation is not the optimum detection for CE-OFDM signal, as it does not comply with the maximum-likelihood (ML) criterion [9]. CE-OFDM is substantially obtained by applying a nonlinear transformation (i.e., the phase modulation) to an OFDM signal. It is known that the ML detection of nonlinearly transformed 
OFDM signals is intractable from a computational viewpoint. ARC detector simply performs a nonlinear compensation of phase modulation. Such combination of nonlinear operations, imposed to the original OFDM signal, involves some critical issues due to the presence of frequency-selective channel response and additive background noise. The most significant is related to phase wrapping. The arctangent function provides $\hat{\phi}(n) \in[-\pi, \pi]$. If $\phi(t)$ in (5) is highly fluctuating, phase wrapping occurs at the output of phase demodulator, resulting in burst errors at the output of QAM demodulator. As mentioned in [7] and [23], the oversampling of the transmitted OFDM sequence is generally an efficient countermeasure against phase wrapping. For a fixed $N$, the dynamic range of the real-valued OFDM signal modulating the carrier phase is limited by oversampling (see [24] for a detailed theoretical analysis applied to generic OFDM signals) and thus occasional jumps at the output of phase demodulator occur with less likelihood. It is worth mentioning here that increasing the oversampling factor $F_{O V}$ increases $N_{D F T}$ and the computational complexity of TX/RX chain without significant performance improvement. The minimum value for the oversampling factor is 2, but, in general, $F_{o v}=4$ and $F_{o v}=8$ are advisable in order to obtain good BER performance thanks to diminishing phase wrapping [7]. Normally, phase unwrapper is employed after the arctangent demodulator for low values of $F_{o v}$, higher modulation order and higher modulation indexes [25] (typically: $2 \pi h>0.7$ ). Phase unwrapping solves the wrapping issues at high $S N R$ regimes, whereas in noise dominant environments phase unwrapping errors (cycle slips) can cause severe performance degradation. Channel coding used in conjunction with interleaving is effective against burst errors and improves system performance, as shown in [25]. Moreover, authors in [25] indicate that phase unwrapper results in a reduced coding gain and, thus, avoid phase unwrapping for applications requiring high data rates obtained with robust channel codes.

The practical implementation of the ARC demodulator is not trivial as remarked in [22] because the computation of the arctangent function can require up to 12 CORDIC iterations to attain 0.1 degree accuracy. The alternate solution relies on accurate Look-Up-Table (LUT)-based implementation that is expensive in terms of storage space. Solutions chraracterized by lower complexity have been proposed in [22]. These solutions calculates the Taylor series expansion of received in-phase and quadrature components of the baseband exponentially-modulated CE-OFDM signal as,

$$
e^{j \phi(t)}=\cos (\phi(t))+j \sin (\phi(t))=I(t)+j Q(t)
$$

For low modulation indexes, higher order terms in Taylor series expansion are neglected, and $I(t)$ and $Q(t)$ can be expressed as follows,

$$
\begin{gathered}
I(t)=\sum_{n=0}^{+\infty}(-1)^{n} \frac{[\phi(t)]^{2 n}}{2 n !} \approx 1-\frac{[\phi(t)]^{2}}{2} \\
Q(t)=\sum_{n=0}^{+\infty}(-1)^{n} \frac{[\phi(t)]^{2 n+1}}{(2 n+1) !} \approx \phi(t)-\frac{[\phi(t)]^{3}}{3 !}
\end{gathered}
$$

From (10) and (11), two simplified receiver schemes are derived in [22]. Using (7), the first one is the Basic Linear Receiver (BLR), works as,

$$
\hat{X}_{n} \approx \frac{Q_{n}^{\rho}}{2 \pi h \Gamma}, \quad n=0,1, \cdots, N_{D F T}-1
$$

and the second one is the Enhanced Receiver (ER),

$$
\hat{X}_{n} \approx \frac{Q_{n}^{\rho}\left[2-I_{n}^{\rho}\right]}{2 \pi h \Gamma}, n=0,1, \cdots, N_{D F T}-1
$$

The BLR effectively works only for low modulation indexes, i.e., $2 \pi h \leqslant 0.5$, while the ER generally yields good performance for moderate modulation indexes $0.5 \leqslant 2 \pi h \leqslant$ 0.7 , for which the cubic term of the Taylor series expansion becomes significant. BLR and ER eliminate the need for phase unwrapper and can work with reduced oversampling factor. Moreover, such simplified detection schemes can even improve BER performance w.r.t. the ARC detector, in particular, when low-rate channel coding is introduced in the transmission system. However, the impact of phase noise is critical with these simplified receivers because the behaviour of phase noise at the output of the nonlinear phase demodulation is not the same as that in ARC detector. Assuming that only phase noise is present in the link, the output of the BLR can be derived as follows,

$$
\hat{X}_{n} \approx \frac{\phi_{n}\left[2-\psi_{n}^{2}\right]-2 \psi_{n}^{3}}{4 \pi h \Gamma}, n=0,1, \cdots, N_{D F T}-1
$$

where $\phi_{n}$ and $\psi_{n}$ are the $n^{\text {th }}$ sample of the phase-modulating signal and phase noise, respectively. It can be seen from (14) that the existence of multiplicative phase noise term $\psi_{n}$ together with $\phi_{n}$ in the estimated sample $\hat{X}_{n}$. For what concerns ER detector, it considers higher order terms of Taylor series for which multiplicative noise terms exist, as shown in the following,

$$
\begin{array}{r}
\hat{X}_{n} \approx \frac{1}{2 \pi h \Gamma}\left[\phi_{n}+\psi_{n}+\frac{3}{4} \phi_{n}^{2} \psi_{n}+\frac{3}{4} \phi_{n} \psi_{n}^{2}+\frac{1}{4} \phi_{n}^{3}\right]+ \\
-\frac{1}{2 \pi h \Gamma}\left[\frac{5}{6} \phi_{n}^{2} \psi_{n}^{3}+\frac{5}{12} \phi_{n} \psi_{4}^{n}+\frac{1}{12} \psi_{n}^{5}\right], \\
n=0,1, \cdots, N_{D F T}-1
\end{array}
$$

From the equations shown above, it can be concluded that ER is, in principle, more vulnerable to phase noise than BLR. Despite this, in our performance evaluation we shall consider the ER because it clearly outperforms BLR in presence of additive noise and multipath, when reasonably high modulation indexes are employed.

\section{B. Constant-Envelope Single-Carrier OFDM (CE-SC-OFDM)}

The constant-envelope DFT-precoded OFDM has been proposed for multiple access systems in [8]. It is based on the application of DFT precoding (see Fig. 1) on the block of $M$ input $L$-QAM information user symbols, expressed as follows,

$$
\hat{S}_{m}=\sum_{n=0}^{M-1} S_{v} e^{\frac{-j 2 \pi n m}{M}}, m=0,1, \cdots, M-1
$$




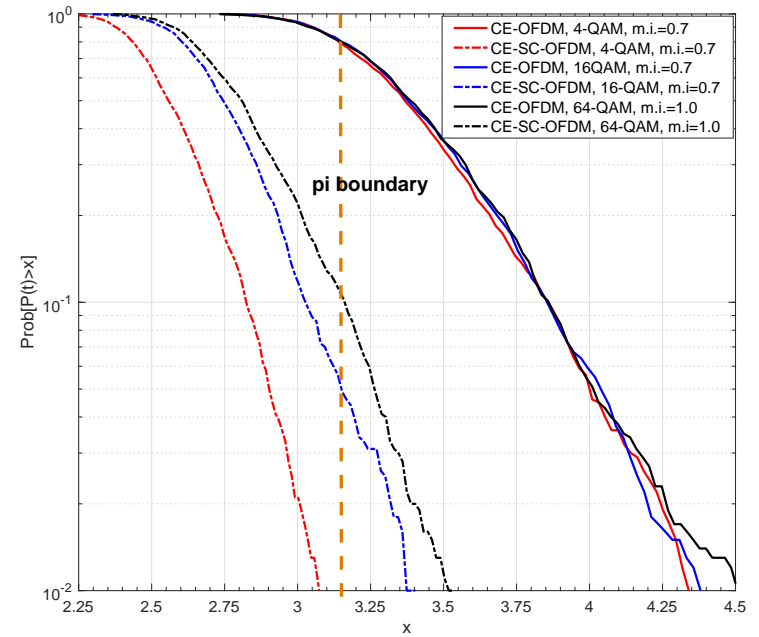

Figure 2. Empirical complementary cumultative distribution function (CCDF) of $P(t)=\max \{|\phi(t)|\}$ for CE-OFDM (solid lines) and CE-SC-OFDM (dash-dotted lines) and different modulation constellations.

The rest of the transmission/detection chain is substantially unmodified as compared to that of CE-OFDM, shown in Fig 1 The nonlinear phase demodulation is implemented with the same methodologies considered for CE-OFDM, namely: ARC, BLR and ER. After the phase demodulation, the DFTbased demultiplexing, and subcarrier demapping, the $M$-point I-DFT decoding recovers the transmitted $L$-QAM information symbols. The theoretical motivation behind CE-SC-OFDM waveform is intrinsically the reduced PAPR of SC-OFDM w.r.t OFDM; thus, the amplitude of real-valued SC-OFDM signal is less fluctuating. This straightforwardly leads to a modulating phase signal $\phi(t)$ characterized by reduced probability of crossing the $\pm \pi$ boundary. Such a behavior is dramatically evidenced in Fig. 2, where the empirical complementary cumulative distribution function (CCDF) of the absolute peak values of $\phi(t)$, namely: $P(t)=\max \{|\phi(t)|\}$, is shown for three different $L-Q A M$ modulation constellations. Thanks to these favourable features, CE-SC-OFDM should be regarded as a valuable candidate waveform for $5 \mathrm{G}$.

\section{The influence of modulation index on CE multicarrier link performance}

The modulation index is perhaps the most critical parameter of CE multicarrier systems that requires detailed analysis. It is possible to analytically evaluate the impact of modulation index on CE multicarrier system performance in AWGN channel only when AWGN becomes addititve at the output of the phase demodulator. As shown in [7], this is verified when:

$$
C N R \triangleq 10 \log _{10}\left(A_{c}^{2} T / N_{0}\right)>10 d B
$$

where $N_{0}$ being the AWGN power spectral density. Under such condition, a lower BER bound on CE-OFDM and CE-SCOFDM for the AWGN case that holds for high SNRs can be computed [7],

$$
B E R \geq 2\left(\frac{\sqrt{L}-1}{\sqrt{L}}\right) Q\left(\sqrt{\frac{(2 \pi h)^{2} 6 \log _{2}(\sqrt{L})}{(L-1)}\left(\frac{E_{b}}{N_{0}}\right)}\right)
$$

In high noise environments, when $C N R$ in (17) is less than $10 \mathrm{~dB}$, we shall notice a considerable BER increase w.r.t. the lower bound of (18) due to nonlinear noise effects that are specific to phase modulated systems.

As far as transmission over flat Rayleigh fading channel is concerned, a lower bound on BER is provided in [7] for CE-OFDM working in high SNR regime,

$$
B E R \geq\left(\frac{\sqrt{L}-1}{\sqrt{L}}\right)\left(1-\sqrt{\frac{\kappa}{1+\kappa}}\right)
$$

where,

$$
\kappa=\frac{(2 \pi h)^{2} 3 \log _{2}(\sqrt{L})}{(L-1)}\left(\frac{\alpha E_{b}}{N_{0}}\right)
$$

where $\alpha$ is the average power attenuation of Rayleigh channel. No closed form analytical BER formulation can be straightforwardly derived for the most relevant technical case of frequency-selective fading channels with FDE. However, with the help of computer simulations system performance can be evaluated. The analytical approximations of (18) and (19), although ignoring threshold effect and burst errors due to phase wrapping, may help readers about the key role played by the angular modulation index in determining the link performance.

The use of low modulation indexes $(2 \pi h \leq 0.5)$ reduces the probability of phase wrapping and, therefore, limits error clusterization. But, as a drawback, such low indexes consistently reduce the destination signal-to-noise ratio and cause BER to increase, as made evident by 18 and (19). On the other hand, higher modulation indexes $2 \pi h \geq 1$ are convenient, when higher order $L-$ QAM constellations are employed. However, an arbitrary increase of $2 \pi h$ introduces unrecoverable error clusterization with consequential error-floor that is also noticed in AWGN channel [7]. Experimental results suggest that lower-order modulations generally work better with modulation indexes lower than 1 whereas spectrally-efficient constellations may consider higher modulation indexes. It should be taken into account in all cases that the modulation index does not only impact on link performance, but also on the bandwidth occupation and spectral efficiency of CE multicarrier transmission, as explained in the next subsection.

When the simplified receiver schemes of [22] are used, the modulation index setting presents some additional tradeoffs. For instance, BLR works well at low modulation index $(2 \pi h \leq 0.5)$ mainly because higher order terms are neglected in computation of the Taylor series expansion. Increasing the modulation index to greater than aforesaid value causes irreducible error floor thanks to distortion created by higher order terms that are no longer negligible. It is worth noticing that CE multicarrier transmission with higher order QAM constellations are more sensitive to the distortion involved by the ignored Taylor series terms. Therefore, higher-order QAM constellations will require lower modulation indexes to work 
with BLR.

On the other hand, ER has been designed in order to reduce the distortion due to the cubic term of the Taylor series. In [22], the variance of the residual cubic term distortion affecting the ER receiver has been explicitly computed as,

$$
\sigma_{c}^{2}=\sum_{k=0}^{N_{D F T}-1} \frac{(2 \pi h)^{6}}{2\left(N_{D F T}\right)^{4}} N_{k}^{\text {distort }}
$$

where $N_{k}^{\text {distort }}$ is the number of subcarriers that produce distortion on the $k^{\text {th }}$ subcarrier (see [22] for further details). It is clear from (21) that modulation indexes higher than 1 are not advisble for ER receiver.

\section{Spectral efficiency of CE multicarrier signals}

The spectral efficiency of CE-OFDM and CE-SC-OFDM transmission expressed in terms of $\mathrm{b} / \mathrm{s} / \mathrm{Hz}$ throughput is given by the following formula [7] [8],

$$
\frac{R_{b}}{W}=\frac{1}{2} \frac{\log _{2}(L)}{\max (2 \pi h, 1)}[b / s / H z]
$$

where $W$ is the bandwidth of the main spectrum lobe. As compared to the spectral efficiency of OFDM and SC-OFDM, we notice a decrease, depending on the modulation index, of at least $50 \%$, when $2 \pi h \leq 1$ is employed. This tradeoff is involved by the real-valued multicarrier phase-modulating signal $\phi(t)$, characterized by double-sided power spectrum. Thus, the $0 \mathrm{~dB}$ PAPR and the augmented frequency diversity are paid in terms of reduced spectral efficiency. However, as clearly shown by simulation results in Section $\mathrm{V}$, the advantages achieved by $\mathrm{CE}$ multicarrier waveforms in terms of link performance and goodput clearly compensate this limitation.

A critical issue concerning CE multicarrier signals is related to the sidelobe spectrum power level. It is shown in [23] that the sidelobe power level of CE-OFDM is considerably higher than that of OFDM counterpart, in particular for $W<f \leq 2 W$. Such a spectral regrowth is consequential to the nonlinear transformation of the multicarrier signals imposed by the phase modulation. The use of post-filtering applied to the transmitted signal in order to reduce the sideband spectrum leakage is not advisable, because it would increase PAPR beyond 0dB. A better solution could be represented by the spectral precoding of the input $L-$ QAM symbols, proposed by Chung in [23]. Spectral precoding dramatically reduces the sidelobe power level of the transmitted CE-OFDM signal by introducing frequency domain correlation among complex QAM symbols without any PAPR increase. In such a case, the tradeoff will be in terms of increased computational burden of the receiver system due to spectral decoding and to a slightly noticeable BER performance degradation w.r.t. the uncoded case.

\section{E. Critical comparison with state-of-the-art multicarrier waveforms}

The waveform design concept shown in [5] and [13] is clearly driven by the necessity of maximum exploition of existing frequency bands in the $s u b-6 \mathrm{GHz}$ domain. Substantially, linear signal processing and filtering are required at subcarrier level in order to reach the expected target. From this viewpoint, FBMC seems the best solution as it provides a multicarrier signal that is regarded as multi-channel signal, where each subcarrier corresponds to a separate channel carrying transmitted information independently. Some of distinguished features of FBMC signal are spectral compactness, efficient utilization of bandwidth as FBMC avoids the cyclic prefix, and is very suitable to occupy unused spectrum portions in cognitive and opportunistic manner. In our opinion, FBMC and similar waveforms are best suited to assure the coexistence of $4 \mathrm{G}$ services in $5 \mathrm{G}$, using the bandwidth portions already occupied by LTE services.

Moving higher in frequency spectrum to the mm-Wave domain, the waveform design requirements vary from those in lower spectrum. The analysis and modeling of mm-Wave urban propagation environments reported in [2] and [26] confirm that the biggest issue that still hinders the exploitation of enormous millimetric bandwidth resources is related to the huge pathloss and shadowing. The high PAPR of state-of-theart multicarrier waveforms require large IBO values to work with saturating amplifiers, hence minimizing the efficiency of RF power amplifier and increasing power consumption. Moreover, the large bandwidth availability (up to $10 \mathrm{GHz}$ in the E-band) allows to relax the severe constraints on spectral efficiency and reduced spectrum leakage. Another key issue is related to phase noise that severely impacts on link performance of high-bit-rate conventional OFDM-derived mm-Wave transmission, while CE multicarrier modulations look overall more resilient to such impairment. From these viewpoints, CE multicarrier waveforms look better tailored to support $5 \mathrm{G}$ broadband mm-Wave applications.

Some noticeable features of "OFDM-inspired" waveforms are also maintained by CE multicarrier counterparts, such as the full-digital signal synthesis obtained by means of IDFT/DFT processing tools and the capability of providing flexible orthogonal multiple access in the downlink. On the other hand, CE-OFDM and CE-SC-OFDM present some weaknesses that can be listed as follows:

- Increased bandwidth occupation or, dually, reduced b/s/Hz throughput;

- Nonlinear arctangent modulation is not trivial to be implemented and introduces phase wrapping and unwanted bursts of errors. Simplified detection schemes effectively work only with low modulation indexes and look more vulnerable to phase noise;

- Orthogonal multiple access cannot be straightforwardly provided in the uplink.

To sum up, CE multicarrier waveforms cannot be regarded as "the solution" to any $5 \mathrm{G}$ broadband transmission; rather just a very efficient solution for some specific applications that may coexist with other waveforms for different application contexts and operating at other frequencies.

\section{The Application Scenario}

\section{A. Scenario description}

As anticipated in section [1] a short-range outdoor picocell downlink scenario is considered in order to assess the effec- 
tiveness of the CE multicarrier waveforms. As mentioned in [27], a large part of wireless traffic is concentrated in hot zones of limited extension. Therefore, an effective method to enhance the quality of experience for the users is to add new nodes like indoor femtocells and outdoor picocells in hierarchical cell structures, which could significantly shorten transmission distances and allow the efficient reuse of radio resources [27]. The coverage of outdoor picocells is limited by antenna heigth and power capabilities. 3GPP standardization group considers a maximum coverage radius for sub-6 Ghz LTEA picocells of $40 \mathrm{~m}$ [28]. If $\mathrm{mm}$-Wave bands are considered for broadband transmission, the coverage radius should be conveniently reduced to $10 \mathrm{~m}$. Such cell size reduction is fully compliant with the trend to densification characterizing $5 \mathrm{G}$ networks. In our scenario, we shall evaluate the link performance and the reachable throughput vs. radial distance in a single picocell. Multi-cell hierarchical scenarios dealing with the issue of inter-cell interference management, like those shown in [27], will be considered in future works.

\section{B. Millimeter-wave RF design}

Our picocell base-station makes use of a Surface Integrated Waveguide (SIW) slot array [29]. This solution has been chosen since it is low cost, w.r.t. classical waveguide solutions, and low profile, w.r.t. reflectors, even at the selected nominal frequency of $73 \mathrm{GHz}$ [30] [31]. SIW are integrated waveguide-like structures fabricated by using two periodic rows of metallic vias connecting the top and the bottom ground planes of a dielectric substrate. They are very promising since they combine most of the advantages of planar printed circuits (compactness, light weight, easiness to fabricate, flexibility, and low cost) and of metallic waveguides (low losses, complete shielding, power handling). Moreover, SIW structures allow integrating active circuits, passive components and radiating elements on the same substrate [32]. For applications in mm-Wave band, multilayer fabrication techniques like LTCC (Low Temperature Co-fired Ceramics) can be conveniently employed for massmarket production [33]. Here, the antenna has been designed to generate a cosecant squared pattern in the elevation plane so to provide an uniform incident power density, for any user position, in the coverage area of almost $40^{\circ}$ [34]. This is shown in Fig. 3 by the ideal mask (blue curve), described by $18+20 * \log _{10}\left[\frac{\sin \theta_{\min }}{\sin \theta}\right]$ with $\theta_{\min } \leq \theta \leq \theta_{\max }$, where $\theta_{\min }=5^{\circ}$ and $\theta_{\max }=40^{\circ}$. Moreover, the antenna shows a half power beam width (HPBW) of $33^{\circ}$ in the azimuthal plane.

Fig. 4 shows the slots and pins layout for the proposed antenna. It can be noted that the antenna is implemented by repeating a basic slot array structure, made by thirteen slots. The substrate used is RT-DUROID $5880\left(\epsilon_{r}=2.2\right.$ and $\tan \delta=0.0009$ ). All the metallic vias have $0.2 \mathrm{~mm}$ diameter and 0.4 pitch size [35] and all the slots are $0.1 \mathrm{~mm}$ wide. For the array radiation pattern synthesis, an alternate projection method has been used by forcing the coefficients of the Schelkunoff polynomial to be symmetrical complex conjugate. Thus, it allows applying the classical method described in [36]. To take into account the mutual coupling between the slots,

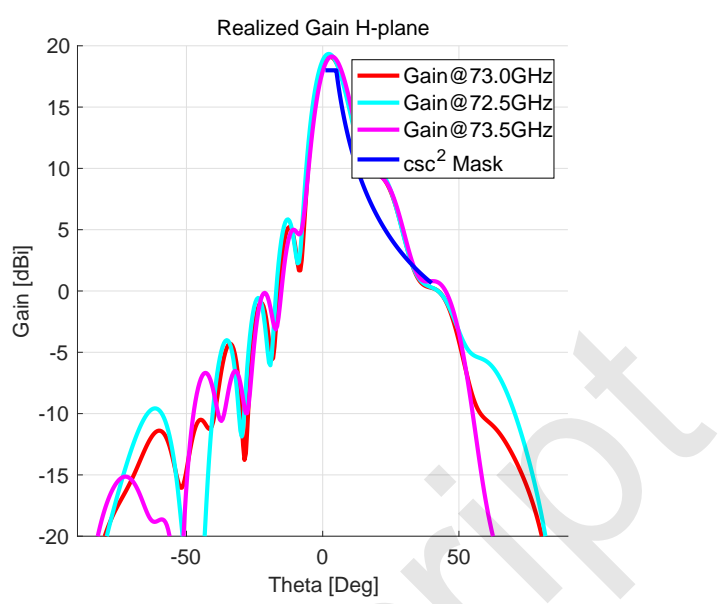

Figure 3. Cosecant squared gain pattern in the elevation plane $\mathrm{xz}$ for the proposed SIW antenna. The pattern is stable above the cosecant squared mask in $1 \mathrm{GHz}$ frequency band around the central frequency of $73 \mathrm{GHz}$.

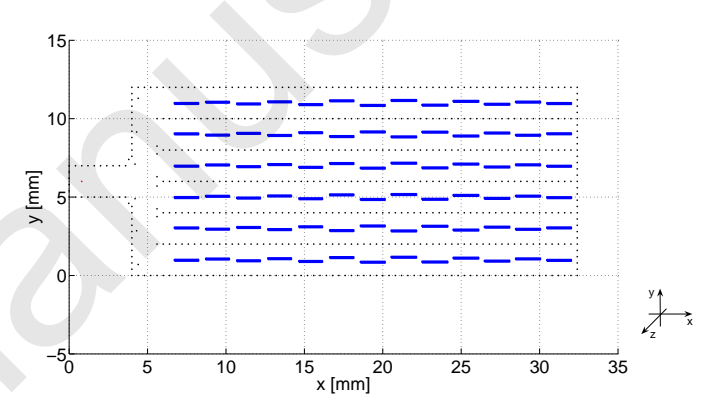

Figure 4. The proposed SIW antenna (35.6x12 mm.).

an iterative method that makes use of a full wave analysis of the entire structure has been applied to determine the optimal length and position of each slot [35]. In Figs. 5 and 6 they are shown the normalized conductance and susceptance associated to each slot respectively, at the beginning and at the end of the iterative process, compared to the theoretical ones. The length and position of each slot with respect to the waveguide axis, shown in Table I correspond to the final values, which give origin to the gain patterns in Fig. 3, It is worth noting that a good similarity of the gain patterns is obtained in $1 \mathrm{GHz}$ bandwidth. The antenna gain is almost $20 \mathrm{dBi}$, corresponding to a radiation efficiency higher than $75 \%$.

\section{Pathloss and shadowing in the E-band}

In the design of mm-Wave transmission systems, the impact on link budget of large-scale propagation phenomena should be carefully assessed. Indeed, pathloss affecting mm-Wave bands is much larger than that measured in sub-6 $\mathrm{GHz}$ bands. The comprehensive link attenuation due to large scale propagation, denoted by $L_{p}$, can be expressed as follows,

$$
L_{p}=P L+L_{O_{2}}+L_{\text {rain }}+\epsilon_{s}(d B),
$$

where $P L$ represents the distance pathloss, $L_{O_{2}}$ represents the oxygen absorption, $L_{\text {rain }}$ denotes the rain attenuation and $\epsilon_{s}$ is the shadowing contribution modeled using a Gaussian random variable with zero mean and standard deviation depending on 
Table I

LENGTH AND OFFSET OF EACH SLOT FOR EVERY WAVEGUIDE

\begin{tabular}{|l|l|l|l|l|l|l|l|l|l|l|l|l|l|}
\hline Slot number & 1 & 2 & 3 & 4 & 5 & 6 & 7 & 8 & 9 & 10 & 11 & 12 & 13 \\
\hline Length $(\mathrm{mm})$. & 1.5947 & 1.5945 & 1.6250 & 1.6211 & 1.6301 & 1.6184 & 1.5875 & 1.5459 & 1.5051 & 1.5103 & 1.4874 & 1.5128 & 1.5306 \\
\hline Offset $(\mathrm{mm})$. & 0.3305 & 0.5196 & 0.7889 & 1.0197 & 1.2519 & 1.6309 & 1.8702 & 1.8412 & 1.2405 & 1.0222 & 0.8231 & 0.4787 & 0.3150 \\
\hline
\end{tabular}

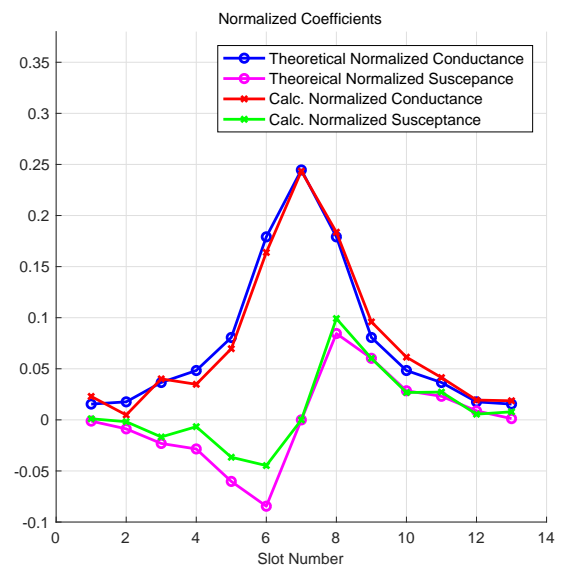

Figure 5. Normalized conductance and susceptance of each slot at the beginning of the iterative process, compared to the theoretical values.

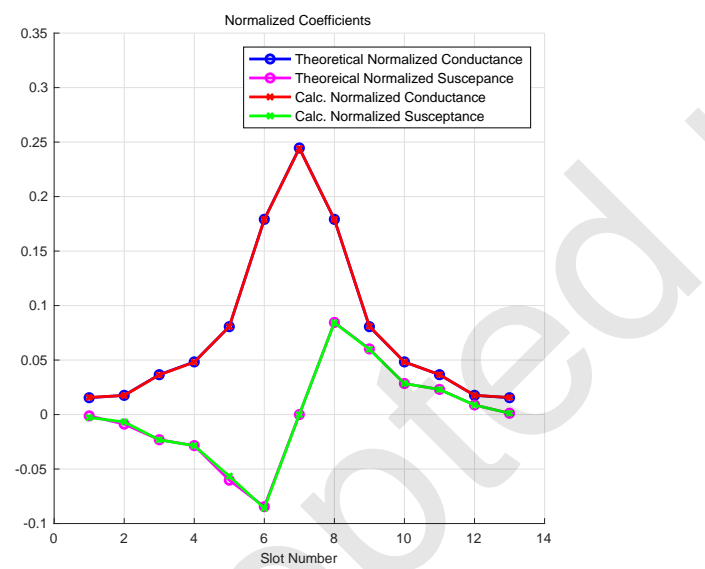

Figure 6. Normalized conductance and susceptance of each slot at the end of the iterative process, compared to the theoretical values.

the specific propagation environment. In Table II the analytical modeling and the parametrization of $P L$ are conveniently summarized (model and numerical parameters are taken from [2]). As far as rain attenuation and oxygen absorption are concerned, their effects in very short-range communication scenarios, considered in this paper, are overall negligible, therefore, $L_{\mathrm{O}_{2}}=L_{\text {rain }} \approx 0 \mathrm{~dB}$. It can easily be noticed from Tab. [1] that the pathloss measured in LOS propagation condition is much more favourable than that measured in the NLOS case. The same happens for the shadow standard deviation, which equals to $5.8 \mathrm{~dB}$ in case of LOS propagation, while the corresponding NLOS value is $8.0 \mathrm{~dB}$ [2].

In Fig. 7 the achievable link signal-to-noise ratio, computed over a bandwidth slot of $500 \mathrm{MHz}$, is plotted vs. distance

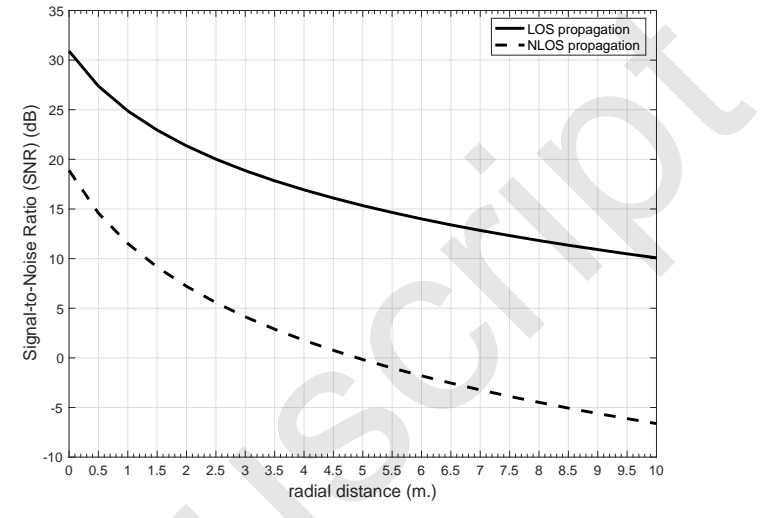

Figure 7. Measured SNR vs. radial distance for the SIW antenna array

for the considered outdoor picocell scenario in case of LOS and NLOS propagation. The transmit antenna is the SIW array detailed in subsection IV-B. The receive antenna of the user terminal is assumed to be isotropic and loseless. The background noise spectral density is assumed equal to -174 $\mathrm{dBm} / \mathrm{Hz}$. The shadow margin has been computed in order to obtain an outage probability at the cell edge equal to $95 \%$ that is acceptable for services like multimedia content delivery and web surfing, typically offered by small outdoor hotspots. The height of the radiating elements and the transmitted power per sector are assumed equal to $3 \mathrm{~m}$. and $12.5 \mathrm{dBm}$, respectively. The latter value represents the saturation power provided by the nonlinear $73 \mathrm{GHz}$ SSPA of [37]. The curves of Fig. 7 clearly show that the occurrence of NLOS propagation corresponds to outage at cell border, as the measured SNR is inferior to $-5 \mathrm{~dB}$ that is well below any acceptable quality threshold. On the other hand, in the LOS case, the SNR at the cell border is around $10 \mathrm{~dB}$ that may represent a reasonable value. The three-state channel model proposed in [2] and detailed in Appendix $\mathrm{A}$ indicates that the considered outdoor picocell scenario belongs to the LOS-dominant propagation region, with a LOS probability at the cell border equal to $86 \%$.

\section{Coded modulation design}

For the baseband section of the picocell transmission system we adopt a design criterion quite similar to that considered in [21]. Substantially, in [21], variable-rate punctured trellis coding has been configured so that largest coding gains are associated to lower order QAM constellations and vice versa. This rate allocation tightly fits with the requirements typical of broadband short-range connectivity, where the highest throughput is expected to be reached very close to the access point, while at longer distances, lower throughput is anyway 
Table II

MODELING AND PARAMETERIZATION OF DISTANCE PATHLOSS FOR $73 \mathrm{GHZ}$ MM-WAVE LINKS (THE LINK DISTANCE $d_{m}$ IS IN METERS)

\begin{tabular}{|l|c|c|}
\hline \multirow{2}{*}{ Analytical model } & \multicolumn{2}{|c|}{ Model parameter values } \\
\cline { 2 - 3 } & NLOS: & LOS: \\
\hline$P L=\alpha+10 \beta \log _{10}\left(d_{m}\right)$ & $\alpha=86.6, \beta=2.45$ & $\alpha=69.8, \beta=2$ \\
\hline
\end{tabular}

guaranteed by fostered coding and modulation arrangements. Trellis coding is no longer the baseline solution in emerging wireless standards, because turbo and Low-Density Parity Check (LDPC) coding show performance closer to the theoretical bounds. In the present work, we have chosen as baseline solution punctured turbo coding, as considered in LTE and LTE-A. Modulation constellations and turbo coding rates employed for our assessments, namely: 4-QAM with $1 / 2$ rate, 16-QAM with 3/4 rate and 64-QAM with $5 / 6$ rate, have been taken by [38], ch.11, sect. 11.3.4.2, pp. 298. Turbo coding will be then compared with LDPC that is currently under consideration for $5 \mathrm{G}$ systems thanks to the lower complexity of decoding operations, noticed, in particular for higher code rates [39].

\section{Simulation Results and Analysis}

\section{A. Simulation strategy}

In order to assess the investigated $\mathrm{CE}$ multicarrier waveforms in the outdoor picocell scenario, we evaluate the performance with different series of results, obtained by means of MATLAB simulation trials of the end-to-end transmission system shown in the block diagram of Fig 1.

The first series, discussed in subsection $\mathrm{V}-\mathrm{C}$, will focus on the mere link performance evaluation in terms of "raw" uncoded BER vs. per-bit signal-to-noise ratio $\frac{E_{b}}{N_{0}}$, attained by CE-OFDM and CE-SC-OFDM waveforms and compared with state-of-the-art OFDM and SC-OFDM. We have not considered the comparison with FBMC, GFDM and UFMC, because the link performance provided by these OFDMderived techniques in the presence of frequency-selective fading is comparable with that of the originating multicarrier techniques [13]; therefore such comparison would have been redundant. Similar considerations can be drawn also for the impact of nonlinear amplification. Without channel coding, it is easier to observe the distortion effects and to compare the simulated BER curves with the analytical lower bounds. The most significant link performance degradation factors will be under-study in this work, namely: frequencyselective multipath propagation, nonlinear distortions due to saturating power amplifiers and phase noise. The effect of nonlinear amplifications and the impact of phase noise will be dealt in separate plots for the sake of clarity and readability of the numerical results. The ideal knowledge of Channel State Information (CSI) has been assumed for all performed simulation trials, while Minimum Mean Square Error (MMSE) based frequency-domain equalization is adopted at receivers of all considered waveform designs. For the sake of clarity, we are not presenting results with BLR detector as BLR shows inferior performance and only works at low modulation indexes as compared to ER and ARC detectors.
The second series of simulation results, detailed and discussed in subsection $\mathrm{V}-\mathrm{D}$, are aimed at providing an assessment of the radial coverage of the picocell downlink. In other words, we shall show some curves plotting the BER attained by the different evaluated waveforms vs. the radial distance of the user terminals from an immobile access point. In these simulation trials, the SNR values vs. radial distance, shown in Fig. 7, are sent as input to the MATLAB simulator. Then, we shall map the achieved BER results into goodput values, obtained by the analytical bound of [40] computed by Padhye et. al. for terrestrial TCP/IP networks. In order to test the coverage reached by the different assessed waveforms in realistic manner, we have considered the simultaneous presence of all the impairments, as mentioned earlier, affecting the real transmission, namely: LOS multipath propagation (NLOS is regarded as outage, as claimed in subsection IV-C), nonlinear amplifier distortion and phase noise. As these simulations trials aim to be as much realistic as possible, channel coding has been introduced; in particular, turbo and LDPC coding designed according to the guidelines illustrated in subsection IV-D

\section{B. Channel modeling and simulation parameters}

As the presence of the LOS component is a dominant condition in short-range networking scenarios like outdoor picocells [41], we shall consider a quasi-static $73 \mathrm{GHz}$ Ricean clustered multipath channel, whose impulse response has been generated based upon the guidelines shown in [42] and [43]. The Rice factor has been assumed equal to $10 \mathrm{~dB}$, according to what stated in [43].

As far as the nonlinear amplifier is concerned, the $P_{i n}$ vs. $P_{\text {out }}$ points of the real SSPA power characteristic taken by [37] have been fitted by a $7^{t h}$-order polynomial model [44] in order to reach the best match between the real and the simulated characteristic. Input power backoff $I B O=15 \mathrm{~dB}$ is required by this device to resort to linear amplification. The corresponding output backoff $(O B O)$ equals to $7.5 \mathrm{~dB}$.

Phase noise SSB power spectral density (PSD) of the 73 $\mathrm{GHz}$ Voltage Controlled Oscillator (VCO), supplied by the Rosa MWave Solutions, LLC (Danver, MA) [45], has been considered in our simulations in order to provide realistic phase noise parameterization. The related PSD values (expressed in $\mathrm{dBc} / \mathrm{Hz}$ ) are shown in Tab. III

The simulation parameters related to the CE-OFDM transmission system are finally reported in Tab. [V] along with the specification about their usage in the concerned simulation series. Regarding the analytical computation of TCP/IP link goodput of [40], the numerical parameterization of such a formulation has been reported in Tab $\mathrm{V}$. 
Table III

SSB PHASE NOISE VALUES TAKEN BY THE COMMERCIAL VCO COMPONENT OF [45]

\begin{tabular}{|l|l|}
\hline frequency offset & SSB value \\
\hline $100 \mathrm{KHz}$ & $-84 \mathrm{dBc} / \mathrm{Hz}$ \\
\hline $1 \mathrm{MHz}$ & $-105 \mathrm{dBc} / \mathrm{Hz}$ \\
\hline $10 \mathrm{MHz}$ & $-125 \mathrm{dBc} / \mathrm{Hz}$ \\
\hline
\end{tabular}

Table IV

LINK PERFORMANCE EVALUATION SIMULATION PARAMETERS

\begin{tabular}{|l|l|}
\hline Parameter & Numerical value \\
\hline TX bandwidth & $500 \mathrm{MHz}$ \\
\hline Modulation constellations & $4-\mathrm{QAM}, 16-\mathrm{QAM}$, 64-QAM \\
\hline Modulation index & $0.2 \leq 2 \pi h \leq 1$ \\
\hline $\begin{array}{l}\text { Channel coding } \\
\left(2^{\text {nd }} \text { series only) }\right.\end{array}$ & Punctured turbo, LDPC coding \\
\hline $\begin{array}{l}\text { Coded modulation } \\
\left(2^{\text {nd }} \text { series only) }\right.\end{array}$ & $\begin{array}{l}1 / 2 \text {-coded 4-QAM, 3/4-coded 16-QAM, } \\
5 / 6-\text { coded 64-QAM }\end{array}$ \\
\hline$N_{D F T}$ & 1024 \\
\hline $\begin{array}{l}F_{\text {Ov }} \\
\text { (CE multi carrier only) }\end{array}$ & 4 \\
\hline CP length (symbols) & 150 \\
\hline CE multicarrier RX & ARC, ER \\
\hline
\end{tabular}

\section{Link performance evaluation}

In the first set of link performance, the impact of nonlinear distortions are analysed in the system for LOS propagation multipath channel, shown in Fig. 8, 9 and 10 Modulation indexes for CE-multicarrier systems are chosen through adhoc offline simulations that are not reported here for sake of brevity. We would like to emphasize about $0 \mathrm{~dB}$ input power backoff requirement for considered CE multicarrier systems, whereas $I B O=15 \mathrm{~dB}$ is applied to OFDM transmission using all the considered modulation formats, in order to avoid an irreducible error-floor. Such high value of IBO is also considered for SC-OFDM systems with 16-QAM and 64QAM. Moreover, SC-OFDM with 4-QAM is characterized by low PAPR and does not require IBO for its operation. The link performance improvement achieved by CE-OFDM and CE-SC-OFDM against the conventional multicarrier counterparts is far-reaching, accounted in tenths of $\mathrm{dB}$ in most of the analysed cases. This is not only due to the better exploitation of available power resources but also because of intrinsic resilience of $\mathrm{CE}$ multicarrier waveforms against frequency-selective multipath fading. Between CE-OFDM and CE-SC-OFDM, better performance is attained by the latter, as expected, thanks to the reduced envelope fluctuation causing error clustering, as mentioned in Section ARC detector exploits higher modulation indexes that are clearly advantageous, in particular when higher-order symbol

Table V

NUMERICAL PARAMETERS FOR ANALYTICAL GOODPUT COMPUTATION

\begin{tabular}{|l|l|}
\hline Parameter & Numerical value \\
\hline Frame length (bytes) & 1024 \\
\hline Maximum congestion window size (frames) & 1 \\
\hline Number of ACK-acknowledged packets & 2 \\
\hline Time out (related to Round-Trip Time) & $4 \times R T T$ \\
\hline
\end{tabular}

constellations are considered. It is worth noticing that the BER curves provided by CE-OFDM and CE-SC-OFDM using the ARC detector approach the theoretical AWGN bound of (18). On the other hand, the simplified ER scheme of [22] fairly works with low modulation index for higher order of modulation constellations as seen in Fig. 9 and 10, We can say that ER detector can support 64-QAM modulation with modulation index of 0.2 rad, whereas ER can support a modulation index as high as that of ARC detector only when CE-SC-OFDM with 4-QAM is considered (i.e., $0.7 \mathrm{rad}$ ). It can seen in Fig. 8, ER outperforms ARC because the former effectively overcomes the problematic issue of error clustering due to phase wrapping/unwrapping.

In the second share of link performance, we analyse the impact of phase noise on link performance evaluated in Fig. 11, 12 and 13, where we assume linear amplification. The BER values obtained by simulations performed with the noisy oscillator of [45] are compared with that obtained by the transmission system working with ideal oscillator. The robustness of $\mathrm{CE}$ multicarrier waveforms against phase noise effects is evident when ARC detector is adopted, in particular when 4-QAM and 16-QAM symbol constellations are used for data transmission. It is noticed in Fig. 11 and 12 that the related BER curves tightly approach the ones that are obtained by the simulations run with the ideal oscillator. A valuable degree of resilience is also provided by SC-OFDM, thanks to its well-recognized lower sensitivity to frequency and phase offsets [3]. Unlike CE-SC-OFDM, CE-OFDM can be significantly impaired by phase noise, for instance in Fig. 13 where a residual error-floor lower than $10^{-6}$ is noticed for 64-QAM CE-OFDM using the ARC detector. More importantly, low complex ER detector with CE-SC-OFDM provides robustness against phase noise, whereas CE-OFDM using the ER detector is prone to phase noise, especially when higher modulation order is considered. It has now become evident from above analyses that CE-SC-OFDM provides robustness against phase drifts due to nonlinear noise effects and nonideal noisy oscillators in mm-Wave multipath channels.

\section{Coverage analysis in the outdoor picocell application scenario}

The RF link budget analysis, shown in subsections IV-B and IV-C, lays foundation for the coverage analysis in the outdoor picocell scenario, described in subsection IV-A In Fig. 14, radial coverage from access point has been shown for the different assessed waveforms with pico-cell radius of $10 \mathrm{~m}$. is considered. As mentioned in subsection IV-D the performance comparison between turbo and LDPC coding systems will be shown. For such analysis, CE-OFDM and CE-SC-OFDM with only ARC detector are considered becuase of the resilient characteristics against frequency selective fading and phase noise, noticed in the generality of the performed simulation trials. We assume a BER threshold of $10^{-5}$ as the maximum acceptable value in order to achieve a satisfactory QoS. Figs. 14(a), related to turbo coding, and 14(b), related to LDPC coding, overall show a dramatic performance improvement attained by $\mathrm{CE}$ multicarrier waveforms. BER measured for CE-OFDM and 


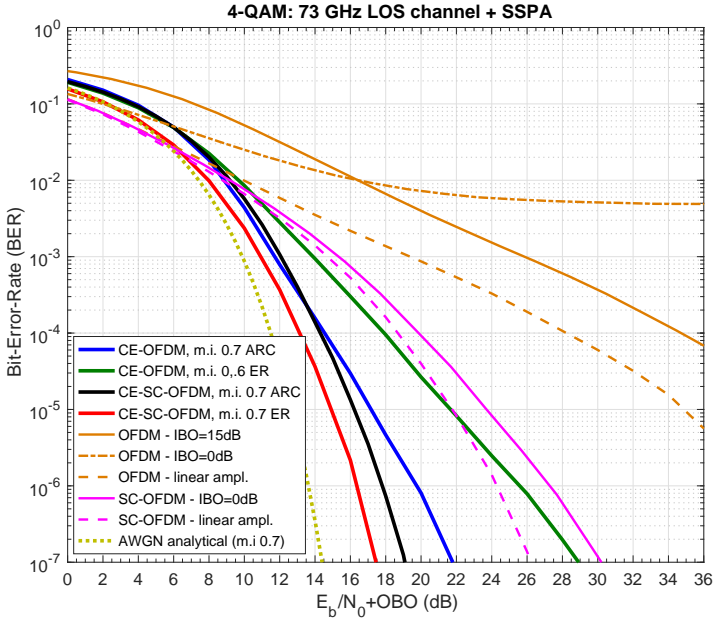

Figure 8. BER vs. $E_{b} / N_{0}$ for CE-OFDM, CE-SC-OFDM, OFDM and SCOFDM: $73 \mathrm{GHz}$ LOS channel, nonlinear amplifier and ideal oscillator, 4 QAM digital modulation.

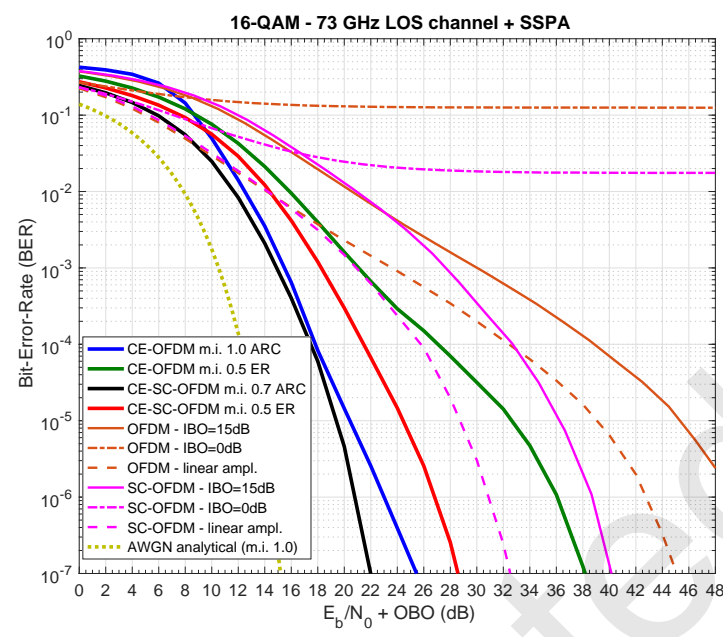

Figure 9. BER vs. $E_{b} / N_{0}$ for CE-OFDM, CE-SC-OFDM, OFDM and SCOFDM: $73 \mathrm{GHz}$ LOS channel, nonlinear amplifier and ideal oscillator, 16QAM digital modulation.

CE-SC-OFDM with 4-QAM modulation and 1/2-rate coding (both turbo and LDPC) is much lower than $10^{-5}$ through the overall cell extension and, therefore, not shown in the plots. Clearly, CE-SC-OFDM performs better than CE-OFDM and claims capacity as well as coverage in pico-cell downlink scenarios. For instance, CE-SC-OFDM with turbo-coded 16QAM attains BER always less than $10^{-5}$ throughout the picocell radius, while the corresponding CE-OFDM transmission trespasses the $10^{-5}$ threshold at a radial distance of about 7.7 $\mathrm{m}$. On the other hand, conventional multicarrier transmission systems are severely impaired by multipath fading, phase noise and, mostly, by the IBO imposed by nonlinear amplification. Higher order turbo-coded M-QAM modulations are capable of providing acceptable BERs only when the user terminal is very close to access point. Only OFDM/SC-OFDM with turbo-coded 4-QAM and SC-OFDM with LDPC-coded 4QAM seem to be competitive for longer distances, but paying

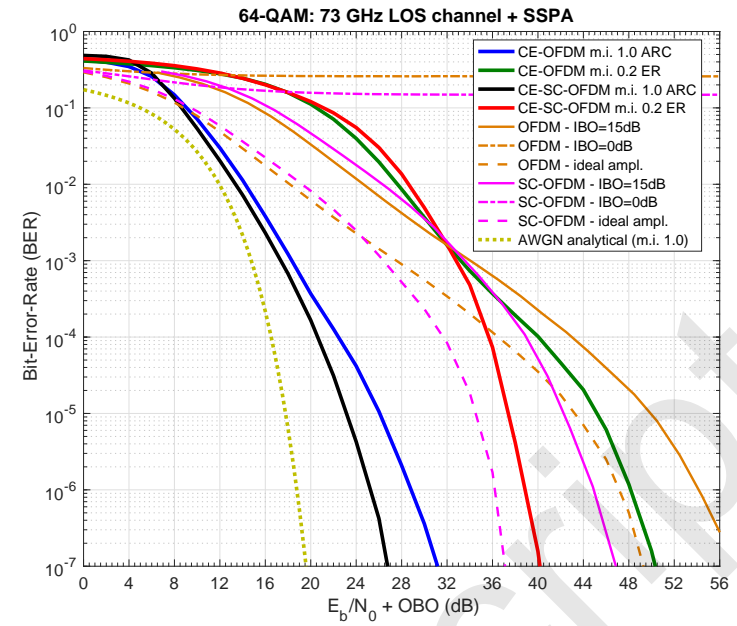

Figure 10. BER vs. $E_{b} / N_{0}$ for CE-OFDM, CE-SC-OFDM, OFDM and SC-OFDM: $73 \mathrm{GHz}$ LOS channel, nonlinear amplifier and ideal oscillator, 64-QAM digital modulation.

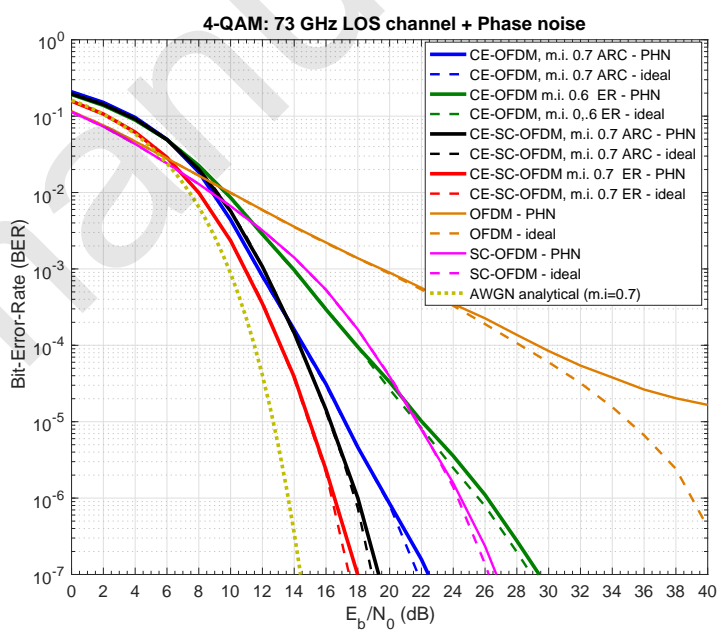

Figure 11. BER vs. $E_{b} / N_{0}$ for CE-OFDM, CE-SC-OFDM, OFDM and SCOFDM: $73 \mathrm{GHz}$ LOS channel, ideal amplification and phase noise, 4-QAM digital modulation.

the price of a limited spectral efficiency. The performance comparison between turbo and LDPC coding applied to CE multicarrier waveforms clearly looks in favor of punctured turbo coding, at least in the considered mmWave scenario, characterized by clustered fading, phase noise and nonlinear demodulation effects. The only exception is represented by conventional SC-OFDM with 3/4-coded 16-QAM modulation. In such a case, LDPC slightly outperforms turbo coding. Results of Fig. 14(a) and 14(b) substantially confirm the claims of some recent works analysing the candidate techniques for 5G channel coding, like e.g. [46], that rank turbo codes in pole-position, although recognizing to LDPCs unquestionable advantages in terms of reduced decoding complexity. The mapping of BER results shown in Fig 14 into the TCP link goodput provided by the analytical bound of [40], normalized w.r.t. the occupied bandwidth, fully confirms the great capacity improvement yielded by CE multicarrier modulations, as 


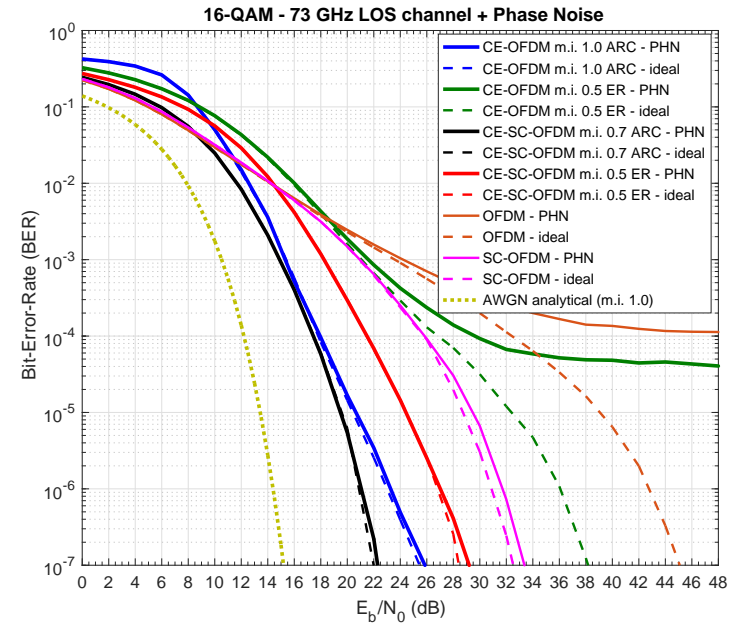

Figure 12. BER vs. $E_{b} / N_{0}$ for CE-OFDM, CE-SC-OFDM, OFDM and SCOFDM: $73 \mathrm{GHz}$ LOS channel, ideal amplification and phase noise, 16-QAM digital modulation.

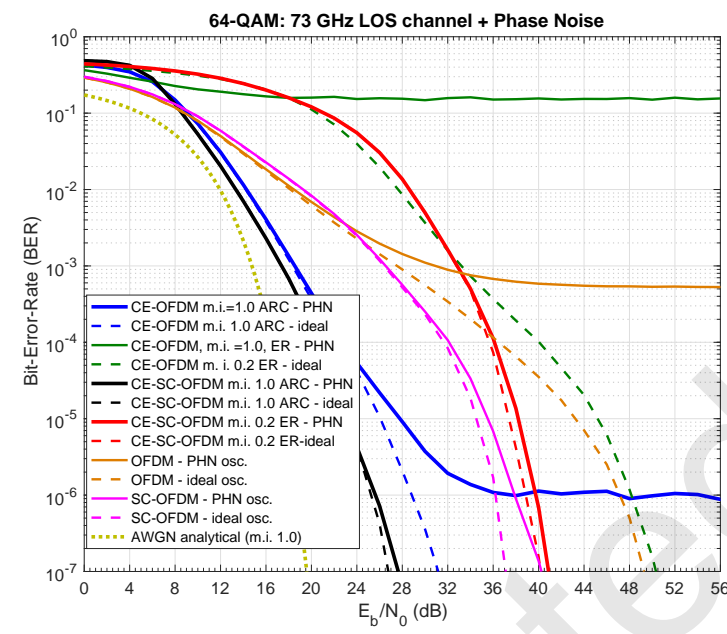

Figure 13. BER vs. $E_{b} / N_{0}$ for CE-OFDM, CE-SC-OFDM, OFDM and SCOFDM: $73 \mathrm{GHz}$ LOS channel, ideal amplification and phase noise, 64-QAM digital modulation.

compared to conventional solutions. Such results are shown in Figs. 15(a) for turbo coding and 15(b) for LDPC coding. SCOFDM can reach the highest goodput $(2.56 \mathrm{~b} / \mathrm{s} / \mathrm{Hz})$ thanks to 16-QAM modulation and 3/4-rate coding, but only for $d=0 \mathrm{~m}$ (turbo coding) and $d=0.5 \mathrm{~m}$ (LDPC coding). As the distance from access point increases, the BER performance drastically degrades for conventional multicarrier techniques. Robust 1/2coded 4-QAM modulation can meet QoS requirements, but only for $d \leq 7 \mathrm{~m}$. On the other hand, CE-SC-OFDM can provide $1.28 \mathrm{~b} / \mathrm{s} / \mathrm{Hz}$ throughout the picocell coverage with $16-$ QAM modulation and 3/4 punctured turbo coding, achieving a $50.23 \%$ gain w.r.t. the conventional multicarrier solutions. The same goodput is reached at shorter distances by 16QAM-modulated CE-OFDM, using either turbo or LDPC coding, and by CE-SC-OFDM using LDPC coding. The highest goodput attained by $\mathrm{CE}$ multicarrier techniques (2.13 $\mathrm{b} / \mathrm{s} / \mathrm{Hz}$ ) can be obtained for $d \leq 2 \mathrm{~m}$. by CE-SC-OFDM with 5/6-LDPC coded 64-QAM modulation. Thanks to the higher modulation index supported, CE-SC-OFDM with turbocoded 64-QAM modulation achieves slightly lower goodput $(1.78 \mathrm{~b} / \mathrm{s} / \mathrm{Hz})$, but at longer distances $(d \leq 5 \mathrm{~m})$. From these results, the broadband coverage is guaranteed by $\mathrm{CE}$ multicarrier waveforms for up to $50 \%$ of the picocell area. It should be noticed that the availability of such a goodput is subjected to the link availability considered to compute the shadow margin (i.e., 95\%) and by the probability of LOS state that varies with the radial distance, computed according to the analytical formulation reported in [2] and shown in Appendix $\mathrm{A}$ Goodput availability, expressed in percentage, against the radial distance is shown in Fig 15 by means of a dedicated ordinate axis. At the picocell border, the plotted goodput values are available for $81.7 \%$ of service time that is satisfactory enough for the applications typically supported by picocells, for instance: multimedia content retrieval and internetworking for leisure.

\section{CONCLUSION}

In this paper, novel waveform candidates are considered for $\mathrm{mm}$-Wave broadband 5G communications, namely: ConstantEnvelope OFDM (CE-OFDM) and Constant-Envelope SCOFDM (CE-SC-OFDM). Their effectiveness for very high data-rate applications in millimeter wave urban channels have been studied first on the basis of theoretical considerations about waveform formation and resilience against mm-Wave propagation impairments and, afterwards, proven by computer simulation, considering a realistically-modeled outdoor picocell downlink scenario. Despite the issue of the 50\% and more throughput reduction due to the increased bandwidth occupation, CE-OFDM and CE-SC-OFDM dramatically outperform the conventional multicarrier counterparts, both in terms of lower BER, and in terms of augmented coverage and network capacity. Future work may concern the performance assessment of the investigated CE multicarrier waveforms when they exploit spectral precoding to reduce sidelobe power level. Another interesting assessment could be related to the impact of nonideal channel estimation on link and capacity performance. From the receiver implementation viewpoint, some research efforts could be spent in order to study computationally-affordable near-optimal detection strategies, for instance considering iterative approaches and/or optimization algorithms able at reducing in effective manner the tremendous size of the ML search space.

\section{APPENDIX A}

THREE-STATE MM-WAVE CHANNEL MODEL

In [2], the probabilistic analyses w.r.t. the link distance $(d)$ for the three-state channel model are represented as follows:

$$
\begin{aligned}
P_{\text {outage }}(d) & =\max \left\{0,1-\exp \left(-a_{\text {out }} d+b_{\text {out }}\right)\right\} \\
P_{\text {LOS }}(d) & =\left\{1-P_{\text {outage }}(d)\right\} \exp \left(-a_{\text {LOS }} d\right) \\
P_{N L O S}(d) & =1-P_{\text {LOS }}(d)-P_{\text {outage }}(d)
\end{aligned}
$$

The parameters used in (24)-(26) have been derived in empirical manner from the NYC measurement campaign, where $1 / a_{L O S}=67.1 \mathrm{~m}, 1 / a_{\text {out }}=30.0 \mathrm{~m}$. and $b_{\text {out }}=5.2$. 


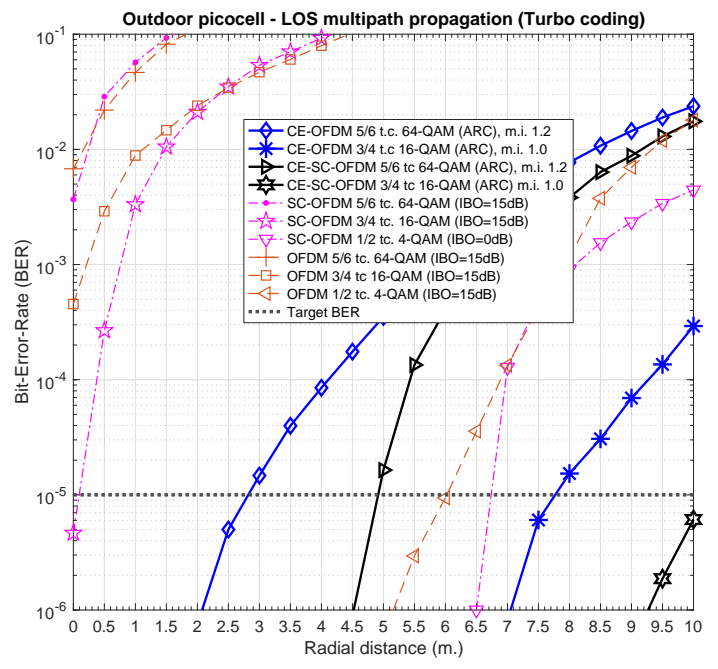

(a)

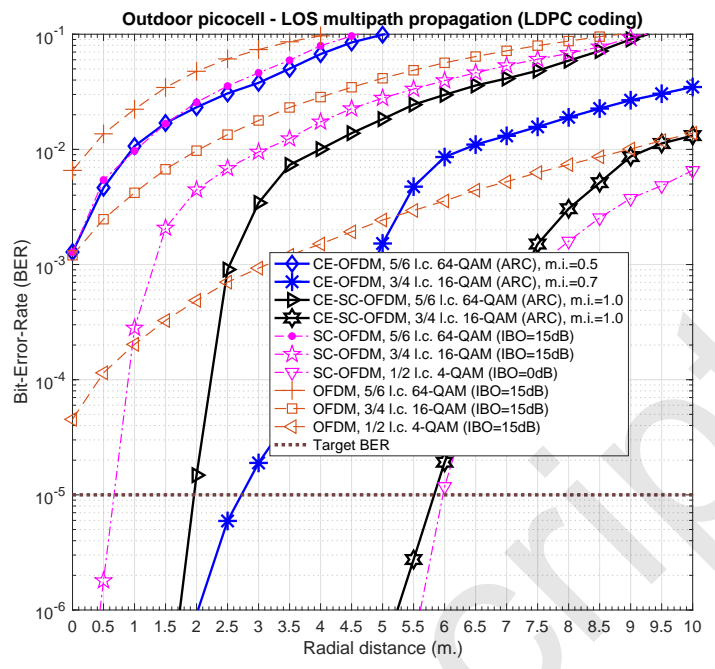

(b)

Figure 14. BER versus radial distance $d$ for the assessed waveforms in the outdoor picocell application scenario under LOS propagation conditions and $95 \%$ link availability: a) punctured turbo coding (t.c.), b) LDPC coding (l.c.)

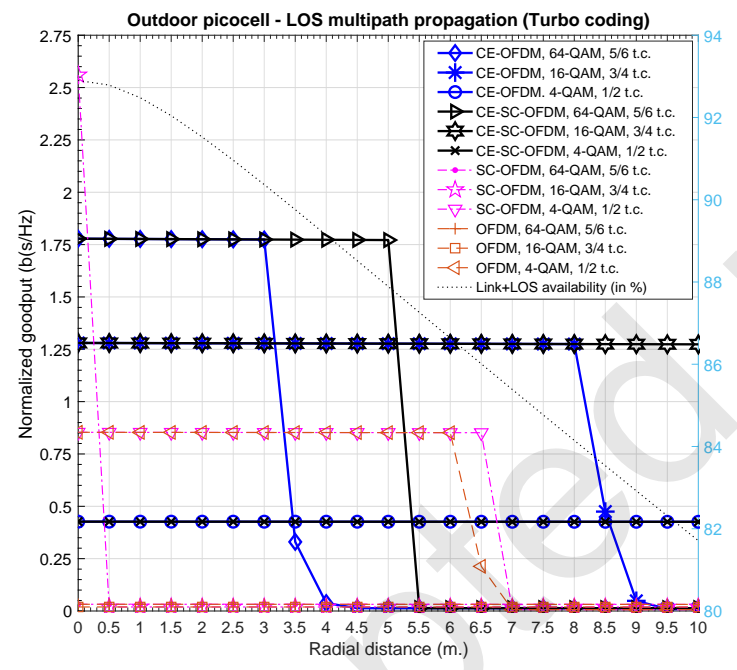

(a)

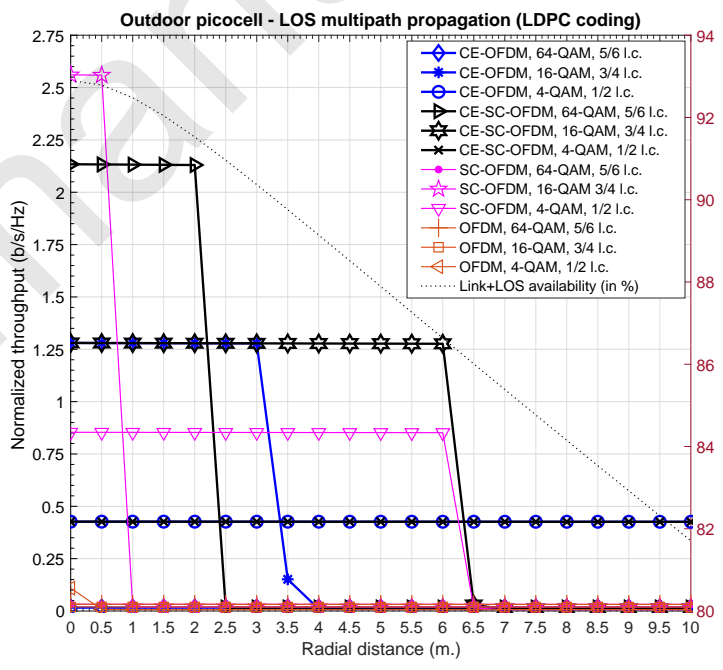

(b)

Figure 15. Normalized goodput versus radial distance $d$ for the different assessed waveforms in the outdoor picocell application scenario under LOS propagation conditions and 95\% link availability: : a) punctured turbo coding (t.c.), b) LDPC coding (l.c.)

\section{REFERENCES}

[1] L. Wei, R. Q. Hu, Y. Qian, and G. Wu, "Key elements to enable millimeter wave communications for $5 \mathrm{G}$ wireless systems," IEEE Wireless Communications, vol. 21, pp. 136-143, December 2014.

[2] M. R. Akdeniz, Y. Liu, M. K. Samimi, S. Sun, S. Rangan, T. S Rappaport, and E. Erkip, "Millimeter Wave Channel Modeling and Cellular Capacity Evaluation," IEEE Journal on Selected Areas in Communications, vol. 32, pp. 1164-1179, June 2014.

[3] H. G. Myung and D. J. Goodman, Single Carrier FDMA. Wiley, 2008.

[4] F. H. Raab, P. Asbeck, S. Cripps, P. B. Kenington, Z. B. Popovic, N. Pothecary, J. F. Sevic, and N. O. Sokal, "Power amplifiers and transmitters for RF and microwave," IEEE Trans. Microw. Theory Tech., vol. 50, pp. 814-826, Mar 2002.

[5] B. Farhang-Boroujeny and H. Moradi, "OFDM Inspired Waveforms for 5G," IEEE Communications Surveys Tutorials, vol. 18, pp. 2474-2492, Fourthquarter 2016.

[6] "5G A Technology Vision," Huawei White Paper, pp. 1-16, 2013. Available at www.huawei.com/ilink/en/download/HW_314849
[7] S. C. Thompson, A. U. Ahmed, J. G. Proakis, J. R. Zeidler, and M. J. Geile, "Constant Envelope OFDM," IEEE Trans. Commun., vol. 56, pp. 1300-1312, August 2008.

[8] R. Mulinde, T. F. Rahman, and C. Sacchi, "Constant-envelope SCFDMA for nonlinear satellite channels," in 2013 IEEE Global Communications Conf. (GLOBECOM), pp. 2939-2944, Dec 2013.

[9] J. Guerreiro, R. Dinis, and P. Montezuma, "On the optimum performance of CE-OFDM schemes in frequency-selective channels," in 2016 International Conference on Telecommunications and Multimedia (TEMU), pp. 1-6, July 2016.

[10] C. Sacchi, E. Cianca, T. Rossi, and M. D. Sanctis, "Analysis and assessment of the effects of phase noise in constant envelope multicarrier satellite transmissions," in 2015 IEEE International Conference on Communications (ICC), pp. 922-927, June 2015.

[11] "5G Waveform Candidates," Rohde \& Schwarz White Paper, pp. 1-60, February 2017. Available at https://www.rohde-schwarz.com/us

[12] Z. Ding, Z. Yang, P. Fan, and H. V. Poor, "On the Performance of NonOrthogonal Multiple Access in 5G Systems with Randomly Deployed 
Users," IEEE Signal Process. Lett., vol. 21, pp. 1501-1505, Dec 2014.

[13] R. Gerzaguet, N. Bartzoudis, L. G. Baltar, V. Berg, J.-B. Doré, D. Kténas, O. Font-Bach, X. Mestre, M. Payaró, M. Färber, and K. Roth, "The 5G candidate waveform race: a comparison of complexity and performance," EURASIP Journal on Wireless Communications and Networking, vol. 2017, p. 13, Jan 2017.

[14] T. Wild, F. Schaich, and Y. Chen, "5G air interface design based on Universal Filtered (UF-)OFDM," in 2014 19th International Conference on Digital Signal Processing, pp. 699-704, Aug 2014.

[15] G. Fettweis, M. Krondorf, and S. Bittner, "GFDM - Generalized Frequency Division Multiplexing," in VTC Spring 2009 - IEEE 69th Vehicular Technology Conference, pp. 1-4, April 2009.

[16] A. G. Armada, "Understanding the effects of phase noise in orthogonal frequency division multiplexing (OFDM)," IEEE Trans. on Broadcasting, vol. 47, pp. 153-159, Jun 2001.

[17] S. Sen and A. Nehorai, "Adaptive OFDM Radar for Target Detection in Multipath Scenarios," IEEE Trans. on Signal Processing, vol. 59, pp. 78-90, Jan 2011.

[18] R. B. Nunes, H. R. de O Rocha, D. A. A. Mello, F. D. Simes, M. E. V. Segatto, and J. A. L. Silva, "Transmission of CE-OFDM Signals Over MMF Links Using Directly Modulated 850-nm VCSELs," IEEE Photonics Technology Letters, vol. 27, pp. 315-318, Feb 2015.

[19] K. M. Rabie, E. Alsusa, A. D. Familua, and L. Cheng, "Constant envelope OFDM transmission over impulsive noise power-line communication channels," in 2015 IEEE Int. Symp. on Power Line Communications and Its Applications (ISPLC), pp. 13-18, March 2015.

[20] R. Magueta, D. Castanheira, A. Silva, R. Dinis, and A. Gameiro, "Iterative space-frequency equalizer for CE-OFDM $\mathrm{mmW}$ based systems," in 2016 IEEE Symp. on Computers and Communication (ISCC), pp. 750755, June 2016

[21] C. Sacchi, T. F. Rahman, N. Bartolomei, S. Morosi, A. Mazzinghi, and F. Ciabini, "Design and Assessment of a CE-OFDM-Based mm-Wave 5G Communication System," in 2016 IEEE Globecom Workshops (GC Wkshps), pp. 1-7, Dec 2016.

[22] A. U. Ahmed and J. R. Zeidler, "Novel Low-Complexity Receivers for Constant Envelope OFDM," IEEE Trans. Signal Process., vol. 63 , pp. 4572-4582, Sept 2015.

[23] C. D. Chung, "Spectral precoding for constant-envelope OFDM," IEEE Trans. Commun., vol. 58, pp. 555-567, February 2010.

[24] M. Sharif, M. Gharavi-Alkhansari, and B. H. Khalaj, "On the peak-toaverage power of OFDM signals based on oversampling," IEEE Trans. Commun., vol. 51, pp. 72-78, Jan 2003.

[25] A. U. Ahmed, S. C. Thompson, and J. R. Zeidler, "Constant Envelope OFDM with Channel Coding," in MILCOM 2006 - 2006 IEEE Military Communications conference, pp. 1-7, Oct 2006.

[26] T. S. Rappaport, G. R. MacCartney, M. K. Samimi, and S. Sun, "Wideband Millimeter-Wave Propagation Measurements and Channel Models for Future Wireless Communication System Design," IEEE Trans. Commun., vol. 63, pp. 3029-3056, Sept 2015.

[27] T. Wang, B. Huang, and Y. Wang, "Bias-based self-organized cell selections for outdoor open-access picocell networks," in 2013 IEEE Wir. Comm. and Networking Conf. (WCNC), pp. 1180-1185, Apr. 2013.

[28] "Further advancements for E-UTRA physical layer aspects," tech. rep., 3GPP Tech. Rep. TR 36.814 v9.0.0, 032010.

[29] L. Yan, W. Hong, G. Hua, J. Chen, K. Wu, and T. Cui, "Simulation and experiment on SIW slot array antennas," IEEE Microwave and Wireless Components Letters, vol. 14, pp. 446-448, Sept 2004.

[30] K. Wu, "Towards system-on-substrate approach for future millimeterwave and photonic wireless applications," in 2006 Asia-Pacific Microwave Conference, pp. 1895-1900, Dec 2006.

[31] S. Cheng, H. Yousef, and H. Kratz, "79 GHz Slot Antennas Based on Substrate Integrated Waveguides (SIW) in a Flexible Printed Circuit Board," IEEE Trans. Antennas Propag., vol. 57, pp. 64-71, Jan 2009.

[32] M. Bozzi, L. Perregrini, K. Wu, and P. P. Arcioni, "Current and future research trends in substrate integrated waveguide technology," Radioengineering, vol. 18, no. 2, pp. 201-209, 2009.

[33] B. J. Chen, T. M. Shen, and R. B. Wu, "Dual-Band Vertically Stacked Laminated Waveguide Filter Design in LTCC Technology," IEEE Trans. Microw. Theory Tech., vol. 57, pp. 1554-1562, June 2009.

[34] M. J. Buckley, "Synthesis of shaped beam antenna patterns using implicitly constrained current elements," IEEE Trans. Antennas Propag., vol. 44, pp. 192-197, Feb 1996.

[35] A. M. N. Bartolomei and A. Freni, "A cosecant square pattern substrate integrated waveguide slot array for radar applications," in 2016 10th European Conf. on Antennas and Propag. (EuCAP), pp. 1-2, April 2016.

[36] R. Compton and R. Collin, Antenna Theory. NY: McGraw-Hill, 1969.
[37] D. Zhao and P. Reynaert, "A $40 \mathrm{~nm}$ CMOS E-Band Transmitter With Compact and Symmetrical Layout Floor-Plans," IEEE Journal of SolidState Circuits, vol. 50, pp. 2560-2571, Nov 2015.

[38] H. Holma and A. Toskala, LTE for UMTS: Evolution to LTE-Advanced. Wiley Publishing, 2nd ed., 2011.

[39] J. Chen, A. Dholakia, E. Eleftheriou, M. P. C. Fossorier, and X.-Y $\mathrm{Hu}$, "Reduced-Complexity Decoding of LDPC Codes," IEEE Trans. Commun., vol. 53, pp. 1288-1299, Aug 2005.

[40] J. Padhye, V. Firoiu, D. F. Towsley, and J. F. Kurose, "Modeling TCP Reno performance: a simple model and its empirical validation," IEEE/ACM Trans. on Networking, vol. 8, pp. 133-145, Apr 2000.

[41] C. Sacchi, T. F. Rahman, I. A. Hemadeh, and M. El-Hajjar, "MillimeterWave Transmission for Small-Cell Backhaul in Dense Urban Environment: a Solution Based on MIMO-OFDM and Space-Time Shift Keying (STSK)," IEEE Access, vol. 5, pp. 4000-4017, 2017.

[42] M. K. Samimi and T. S. Rappaport, "3-D statistical channel model for millimeter-wave outdoor mobile broadband communications," in 2015 IEEE International Conference on Communications (ICC), pp. 24302436, June 2015.

[43] M. K. Samimi and T. S. Rappaport, "Local multipath model parameters for generating 5G millimeter-wave 3GPP-like channel impulse response," in 2016 10th European Conf. on Antennas and Propag. (EuCAP), pp. 1-5, April 2016.

[44] J. Ibanez-Diaz, C. Pantaleon, I. Santamaria, T. Fernandez, and D. Martinez, "Nonlinearity estimation in power amplifiers based on subsampled temporal data," IEEE Trans. Instrum. Meas., vol. 50, pp. 882-887, Aug 2001.

[45] Rosa MWave, 71-76 GHz Receiver Module RxM73WR12, June 2015. Product Brief. Available at https://rosamwave.com/wp-content/uploads/2015/07/RxM73WR12/

[46] R. G. Maunder, "The 5G channel code contenders," AccelerComm White Paper, pp. 1-13, August 2016. Available at https://eprints.soton.ac.uk/399915/

Talha Faizur Rahman is post doctoral researcher at the Department of Electrical and Electronic Engineering (DIEE) of the University of Cagliari (Italy) since April 2018. From March 2017 till March 2018, he was Assistant Professor at Center for Advanced Studies in Telecommunications (CAST) in COMSATS Institute of Information Technology, Islamabad Pakistan. The research interests of Dr. Talha Rahman mainly revolves around wireless communication systems and Internet of Things.

Claudio Sacchi $\left(\mathbf{M}^{\prime} 01-\mathrm{SM}^{\prime} 07\right)$ is assistant professor at the Department of Information Engineering and Computer Science (DISI) of the University of Trento (Italy). In 2011, he was guest editor of the special issue of PROCEEDINGS OF THE IEEE: "Aerospace Communications: History, Trends and Future." In 2015, he was guest editor of the special issue of IEEE COMMUNICATIONS MAGAZINE: "Toward the Space 2.0 Era." The research interests of Dr. Sacchi are mainly focused on mobile and satellite communication systems, multiple access systems and software-defined radios.

Simone Morosi received the Ph.D. degree in information and telecommunication engineering from the University of Florence in 2000. He is currently an Assistant Professor with the Information Engineering Department of the University of Florence. He is an author of more than 100 papers in international journals and conference proceedings. His current research interests focus on wireless communications systems, energy-efficient communications and localization techniques.

Agnese Mazzinghi received the Ph.D. in information engineering from the University of Siena (Italy), in 2010. She is currently a Research Assistant in the Radar and Millimeter Wave Laboratory, University of Florence. Her research interests include numerical and asymptotic methods in electromagnetic antenna problems with a particular attention to the design of radial line slot-array antennas, reflectarrays, electromagnetic band gap structures, and dielectric waveguides.

Nicola Bartolomei received the Ph.D. degree in information engineering from the University of Florence in 2017. He is currently a Postdoctoral Fellow with IETR (Institut d'Electronique et de Tlcommunications de Rennes), University of Rennes 1 . His research interests include the analysis and design of substrate integrated waveguide antennas, multibeam antennas, numerical modeling, and the integration of millimeter-wave systems. 